Research Article

\title{
Construction, Validation, and Visualization of Two Web-Based Nomograms to Predict Overall and Cancer-Specific Survival in Patients with Gastric Cancer and Lung Metastases
}

\author{
Honghong Zheng $\mathbb{D}^{1},{ }^{1}$ Zhehong Li $\mathbb{D}^{2},{ }^{2}$ Jianjun Li $\mathbb{D},{ }^{1}$ Shuai Zheng $\mathbb{D}^{1},{ }^{1}$ and Enhong Zhao $\mathbb{B D}^{1}$ \\ ${ }^{1}$ Department of Gastrointestinal Surgery, Affiliated Hospital of Chengde Medical University, Chengde, China \\ ${ }^{2}$ Department of Orthopedic, Affiliated Hospital of Chengde Medical University, Chengde, China \\ Correspondence should be addressed to Enhong Zhao; zhenghh1215@163.com
}

Received 28 May 2021; Accepted 21 October 2021; Published 1 November 2021

Academic Editor: Nicola Silvestris

Copyright (c) 2021 Honghong Zheng et al. This is an open access article distributed under the Creative Commons Attribution License, which permits unrestricted use, distribution, and reproduction in any medium, provided the original work is properly cited.

\begin{abstract}
Background. The lung is one of the most common sites of metastasis in gastric cancer. Our study developed two nomograms to achieve individualized prediction of overall survival (OS) and cancer-specific survival (CSS) in patients with gastric cancer and lung metastasis (GCLM) to better guide follow-up and planning of subsequent treatment. Methods. We reviewed data of patients diagnosed with GCLM in the Surveillance, Epidemiology, and End Results (SEER) database from 2010 to 2015. The endpoints of the study were the OS and CSS. We used the "caret" package to randomly divide patients into training and validation cohorts in a $7: 3$ ratio. Multivariate Cox regression analysis was performed using univariate Cox regression analysis to confirm the independent prognostic factors. Afterward, we built the OS and CSS nomograms with the "rms" package. Subsequently, we evaluated the two nomograms through calibration curves, receiver operating characteristic (ROC) curves, and decision curve analysis (DCA). Finally, two web-based nomograms were built on the basis of effective nomograms. Results. The OS analysis included 640 patients, and the results of the multivariate Cox regression analysis showed that grade, chemotherapy, and liver metastasis were independent prognostic factors for patients with GCLM. The CSS analysis included 524 patients, and the results of the multivariate Cox regression analysis showed that the independent prognostic factors for patients with GCLM were chemotherapy, liver metastasis, marital status, and tumor site. The ROC curves, calibration curves, and DCA revealed favorable predictive power in the OS and CSS nomograms. We created web-based nomograms for OS (https://zhenghh.shinyapps.io/aclmos/) and CSS (https:// zhenghh.shinyapps.io/aslmcss/). Conclusions. We created two web-based nomograms to predict OS and CSS in patients with GCLM. Both web-based nomograms had satisfactory accuracy and clinical usefulness and may help clinicians make individualized treatment decisions for patients.
\end{abstract}

\section{Introduction}

Gastric cancer (GC) is one of the most common malignant tumors of the gastrointestinal tract, accounting for the third and fifth causes of cancer deaths in men and women worldwide, respectively [1]. According to the 2018 Global Cancer Center statistics [1], there were approximately one million new cases of GC and approximately 780,000 GC-related deaths worldwide. Although radical surgery is currently effective in treating localized GC, recurrence or metastasis still occurs in $25 \%$ to $40 \%$ of patients after surgery [2-4]. According to relevant studies, the lung is a frequent metastatic organ in patients with GC [5], and the incidence of lung metastasis (LM) after GC surgery ranges from $1.3 \%$ to $3.8 \%$ [6-10]. Moreover, there is a lack of mature therapy standards for gastric cancer and lung metastasis (GCLM), and the 5year survival rate of patients with GCLM is $<5 \%$ [11]. At this stage, few studies have reported prognostic factors regarding the survival of patients with GCLM. Therefore, establishing a prediction model for patients with GCLM is clinically significant. 
The treatment of GCLM has been recently diversified [12-15]; however, the poor surgical outcome and complications associated with lung-occupying lesions in patients with GCLM lead to worse prognosis. Kong et al. [16] reported that the median survival of patients with GCLM is only four months. Moreover, studies have shown that the prognostic influences of GCLM generally include tumor histological grade, $\mathrm{T}$ stage, concurrent pulmonary metastases, primary lesions not subjected to surgery, bilateral pulmonary metastases, combined extrapulmonary metastases, and chemotherapy [17]. Regrettably, no studies have combined the relevant variables to assess the prognosis of GCLM.

A nomogram is a simple, multivariate visualization tool in oncology for predicting and quantifying individual patient survival, to aid clinical decision-making and promote precision medicine [18-21]. In addition, the web-based nomogram, also known as "predictive probability web page calculator," is a web page based on Shiny. This nomogram is a product of the electronic era, and the user just has to select the appropriate variable and click "Predict" to draw the probability of occurrence of the corresponding characteristics of patients, which is convenient and more practical [22]. Consequently, we aimed to devise two web-based nomograms to predict the overall survival (OS) and cancerspecific survival (CSS) in patients with GCLM based on the Surveillance, Epidemiology, and End Results (SEER) database.

\section{Materials and Methods}

2.1. Data Source and Inclusion Criteria. In this study, our data were obtained by downloading the SEER $*$ Stat software version 8.3.6. The SEER database is a public database, exempt from medical ethics review, and does not require informed consent. Strict inclusion and exclusion criteria were also developed, and the nadir criteria are listed below. The inclusion criteria were as follows: (I) patients diagnosed with GCLM between 2010 and 2015; (II) demographic variables, including age, race and gender, marital status, and insurance status; and (III) available tumor characteristics, including histological grade, $\mathrm{T}$ stage, $\mathrm{N}$ stage, brain metastasis, bone metastasis, and liver metastasis. The exclusion criterion was incomplete information. Next, we randomized the patients into training (70\%) and validation cohorts $(30 \%)$. In this study, patients in the training and validation cohorts were used to develop and validate the nomograms, respectively.

\subsection{Clinicopathological Factors. Clinicopathological factors} for the following variables were extracted: age ( $<60$ and $\geq 60$ years), race (white, black, and other), sex (female and male), histologic type (adenocarcinoma, signet ring cel1, intestinal type, other), T stage (T1, T2, T3, and T4), N stage (NO, N1, and N3), grade (grade I, grade II, grade III, and grade IV), bone metastasis (yes or no), liver metastasis (yes or no), brain metastasis (yes or no), primary site (cardia, fundus, body, gastric antrum, lesser, greater, other), radiotherapy (yes or no), chemotherapy (yes or no), surgery (yes or no), marital status (yes or no), and insurance (yes or no). OS and CSS were considered endpoint times. OS and CSS were, respectively, defined as the time from diagnosis to death from all causes and the time from cancer diagnosis to death.

2.3. Statistical Analysis. All statistical analyses were performed using the $\mathrm{R}$ software (version 4.0.2). $P$ value $<0.05$ (both sides) was considered statistically significant. We obtained relevant prognostic factors through univariate Cox regression analysis and obtained independent prognostic factors through multivariate Cox regression analysis on the basis of univariate Cox regression analysis. The prognostic nomograms for OS and CSS were created separately using the "rms" package, according to the independent prognostic factors. In addition, ROC curves for the prognostic nomograms were established. The area under the curve (AUC) was used to evaluate the discriminative power of the nomograms. In addition, calibration curves and decision curve analysis (DCA) for nomograms were established. Finally, we divided all patients into high- and low-risk groups according to the median risk score and tested the prognostic value of the nomograms using Kaplan-Meier (KM) analysis.

\section{Results}

3.1. Flowchart. A detailed workflow is shown in Figure 1.

3.2. Characteristics of the Study Population. For the OS analysis, a total of 640 patients were included, 448 patients in the training cohort and the remaining 192 patients in the validation cohort. Among the 640 patients, the number of male patients $(69.69 \%)$ was higher than that of the female patients $(30.31 \%)$. A total of 484 patients $(75.63 \%)$ were white, 75 patients (11.72\%) were black, and 81 patients (12.65\%) were classified as “other." Of these patients, 219 were below 60 years of age and 421 were 60 years old or older. The baseline clinicopathological characteristics of patients in the OS group are shown in Table 1.

A total of 524 patients for the CSS analysis were enrolled; 368 patients were included in the training cohort, and the remaining 156 patients were included in the validation cohort. Of the 524 patients, $69.08 \%$ were male and $30.92 \%$ were female patients. Most of the patients (70.05\%) were classified as white. Finally, 197 patients were below 60 years of age, and 327 patients were 60 years old or older. The baseline clinical pathological characteristics of patients in the CSS group are shown in Table 2.

3.3. Prognostic Factors for Patients with GCLM. For grouping status of OS, the detailed information of patients with GCLM in the OS group is shown in Table 3. Univariate Cox regression analysis demonstrated that grade II, liver metastasis, radiotherapy, and chemotherapy were OS-related prognostic factors. Multivariate Cox regression analysis showed that grade I1l $(P$ value $=0.018$, hazard ratios $(\mathrm{HR})=$ 1.896, 95\% confidence interval $(\mathrm{CI})=1.118-3.214)$, liver 


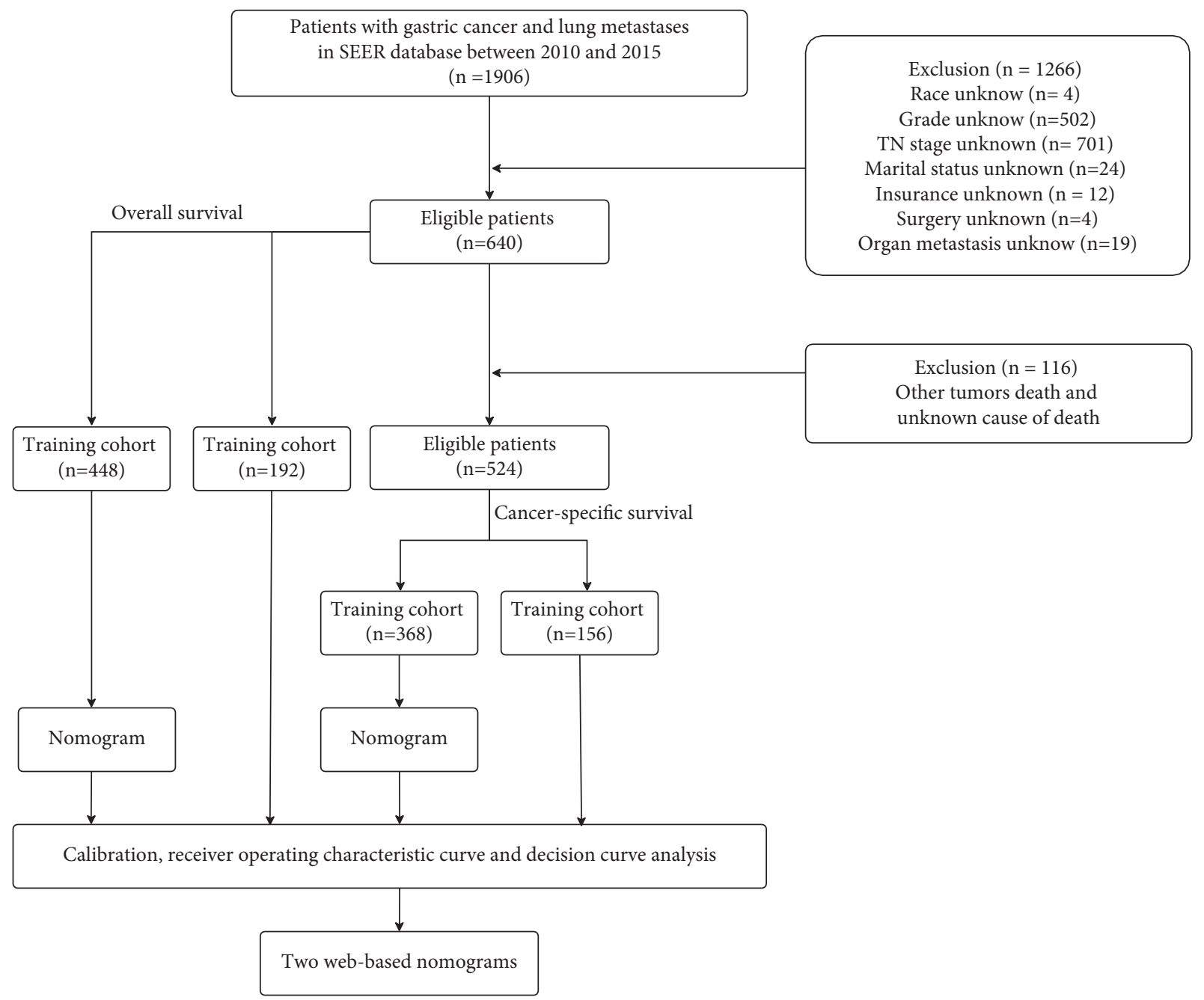

Figure 1: Detailed workflow of study design and analysis.

metastasis $(P \quad$ value $<0.001, \quad \mathrm{HR}=1.440, \quad 95 \%$ $\mathrm{CI}=1.179-1.760)$, and chemotherapy $(P$ value $<0.001$, $\mathrm{HR}=0.292,95 \% \mathrm{CI}=0.235-0.363)$ were independent prognostic factors in patients with GCLM.

For grouping status of CSS, more details of the patients with GCLM in the CSS group are listed in Table 4. Univariate Cox regression analysis revealed that race, T2, liver metastasis, primary site, chemotherapy, and marital status were CSS-related prognostic factors. Multivariate COX regression analysis revealed that liver metastasis $(P$ value $<0.001, \mathrm{HR}=1.524,95 \%$ $\mathrm{CI}=1.217-1.909$ ), primary site (greater, $P$ value $=0.001$, $\mathrm{HR}=2.315,95 \% \mathrm{CI}=1.395-3.814)$, chemotherapy ( $P$ value $<0.001, \mathrm{HR}=0.398,95 \% \mathrm{CI}=0.317-0.501)$, and marital status ( $P$ value $=0.039, \mathrm{HR}=0.778,95 \% \mathrm{CI}=0.629-0.988)$ were independent prognostic factors for GCLM.

3.4. Establishment of Nomogram. Prognostic nomograms of OS were established according to three independent prognostic factors (Figure 2(a)). Prognostic nomograms of CSS were created according to four independent prognostic factors (Figure 2(b)).

\subsection{Verification of Nomogram}

(i) ROC of OS: The AUCs at 3, 6, and 12 months were $0.753,0.799$, and 0.732 , respectively, in the training cohort (Figures 3(a)-3(c)). In the validation cohort, the AUCs at 3, 6, and 12 months were $0.855,0.755$, and 0.686 , respectively (Figures $3(\mathrm{~d})-3(\mathrm{f})$ ). The time-dependent ROC curves revealed that the AUC value fluctuated at approximately 0.8 from one month to 12 months (Figures $3(\mathrm{~g})$ and $3(\mathrm{~h})$ ).

(ii) ROC of CSS: The AUCs at 3, 6, and 12 months were, respectively, $0.820,0.766$, and 0.760 , respectively, in the training cohort (Figures 4(a)-4(c)). The AUCs at 3,6 , and 12 months were separately $0.894,0.764$, and 0.720 , respectively, in the validation cohort (Figures 4(d)-4(f)). The time-dependent ROC curves also demonstrated that the AUC value fluctuated at approximately 0.8 from one month to 12 months (Figures 4(g) and 4(h)).

(iii) Calibration curves: The calibration curves at 3,6, and 12 months for the OS probabilities were in good correspondence with the OS predicted with the 
TABLE 1: Baseline data of clinicopathological characteristics of patients with GCLM in OS group.

\begin{tabular}{|c|c|c|c|c|c|c|}
\hline \multirow[t]{2}{*}{ Variables } & \multicolumn{2}{|c|}{ Total cohort $(N=640)$} & \multicolumn{2}{|c|}{$\begin{array}{c}\text { Training cohort } \\
\quad(N=448)\end{array}$} & \multicolumn{2}{|c|}{$\begin{array}{c}\text { Validation cohort } \\
\quad(N=192)\end{array}$} \\
\hline & $n$ & $\%$ & $n$ & $\%$ & $n$ & $\%$ \\
\hline \multicolumn{7}{|l|}{ Age } \\
\hline$<60$ & 219 & 34.2 & 158 & 35.3 & 61 & 31.8 \\
\hline$\geq 60$ & 421 & 65.8 & 290 & 64.7 & 131 & 68.2 \\
\hline \multicolumn{7}{|l|}{ Race } \\
\hline Black & 75 & 11.7 & 53 & 11.8 & 22 & 11.5 \\
\hline Other & 81 & 12.7 & 51 & 11.4 & 30 & 15.6 \\
\hline White & 484 & 75.6 & 344 & 76.8 & 140 & 72.9 \\
\hline \multicolumn{7}{|l|}{ Sex } \\
\hline Female & 194 & 30.3 & 127 & 28.3 & 67 & 34.9 \\
\hline Male & 446 & 69.7 & 321 & 71.7 & 125 & 65.1 \\
\hline \multicolumn{7}{|l|}{ Histologic type } \\
\hline Adenocarcinoma & 407 & 63.6 & 288 & 64.3 & 119 & 62.0 \\
\hline Signet ring cell & 94 & 14.7 & 66 & 14.7 & 28 & 14.6 \\
\hline Intestinal type & 42 & 6.6 & 26 & 5.8 & 16 & 8.3 \\
\hline Other & 97 & 15.2 & 68 & 15.2 & 29 & 15.1 \\
\hline \multicolumn{7}{|l|}{ T stage } \\
\hline $\mathrm{T} 1$ & 252 & 39.4 & 168 & 37.5 & 84 & 43.8 \\
\hline $\mathrm{T} 2$ & 40 & 6.3 & 28 & 6.3 & 12 & 6.3 \\
\hline $\mathrm{T} 3$ & 147 & 23 & 106 & 23.7 & 41 & 21.4 \\
\hline $\mathrm{T} 4$ & 201 & 31.4 & 146 & 32.6 & 55 & 28.6 \\
\hline \multicolumn{7}{|l|}{$\mathrm{N}$ stage } \\
\hline No & 262 & 40.9 & 185 & 41.3 & 77 & 40.1 \\
\hline N1 & 292 & 45.6 & 198 & 44.2 & 94 & 49.0 \\
\hline N2 & 43 & 6.7 & 35 & 7.8 & 8 & 4.2 \\
\hline N3 & 43 & 6.7 & 30 & 6.7 & 13 & 6.8 \\
\hline \multicolumn{7}{|l|}{ Grade } \\
\hline Grade I & 26 & 4.1 & 16 & 3.6 & 10 & 5.2 \\
\hline Grade II & 178 & 27.8 & 124 & 27.7 & 54 & 28.1 \\
\hline Grade III & 426 & 66.6 & 302 & 67.4 & 124 & 64.6 \\
\hline Grade IV & 10 & 1.6 & 6 & 1.3 & 4 & 2.1 \\
\hline \multicolumn{7}{|l|}{ Bone metastasis } \\
\hline No & 526 & 82.2 & 372 & 83 & 154 & 80.2 \\
\hline Yes & 114 & 17.8 & 76 & 17 & 38 & 19.8 \\
\hline \multicolumn{7}{|l|}{ Liver metastasis } \\
\hline No & 325 & 50.8 & 222 & 49.6 & 103 & 53.6 \\
\hline Yes & 315 & 49.2 & 226 & 50.4 & 89 & 46.4 \\
\hline \multicolumn{7}{|l|}{ Brain metastasis } \\
\hline No & 622 & 97.2 & 436 & 97.3 & 186 & 96.9 \\
\hline Yes & 18 & 2.8 & 12 & 2.7 & 6 & 3.1 \\
\hline \multicolumn{7}{|l|}{ Primary site } \\
\hline Cardia & 291 & 45.5 & 201 & 44.9 & 90 & 46.9 \\
\hline Fundus & 35 & 5.5 & 22 & 4.9 & 13 & 6.8 \\
\hline Body & 44 & 6.9 & 31 & 6.9 & 13 & 6.8 \\
\hline Gastric antrum & 74 & 11.6 & 53 & 11.8 & 21 & 10.9 \\
\hline Lesser & 28 & 4.4 & 21 & 4.7 & 7 & 3.6 \\
\hline Greater & 30 & 4.7 & 22 & 4.9 & 8 & 4.2 \\
\hline Other & 138 & 21.6 & 98 & 21.9 & 40 & 20.8 \\
\hline \multicolumn{7}{|l|}{ Radiotherapy } \\
\hline No & 492 & 76.9 & 348 & 77.7 & 144 & 75.0 \\
\hline Yes & 148 & 23.1 & 100 & 22.3 & 48 & 25.0 \\
\hline Chemotherapy & & & & & & \\
\hline No & 256 & 40 & 179 & 40 & 77 & 40.1 \\
\hline Yes & 384 & 60 & 269 & 60 & 115 & 59.9 \\
\hline Surgery & & & & & & \\
\hline No & 580 & 90.6 & 407 & 90.8 & 173 & 90.1 \\
\hline Yes & 60 & 9.4 & 41 & 9.2 & 19 & 9.9 \\
\hline Marital status & & & & & & \\
\hline No & 255 & 39.8 & 182 & 40.6 & 73 & 38.0 \\
\hline
\end{tabular}


TABle 1: Continued.

\begin{tabular}{|c|c|c|c|c|c|c|}
\hline \multirow[t]{2}{*}{ Variables } & \multicolumn{2}{|c|}{ Total cohort $(N=640)$} & \multicolumn{2}{|c|}{$\begin{array}{l}\text { Training cohort } \\
\quad(N=448)\end{array}$} & \multicolumn{2}{|c|}{$\begin{array}{l}\text { Validation cohort } \\
\qquad(N=192)\end{array}$} \\
\hline & $n$ & $\%$ & $n$ & $\%$ & $n$ & $\%$ \\
\hline Yes & 385 & 60.2 & 266 & 59.4 & 119 & 62.0 \\
\hline \multicolumn{7}{|l|}{ Insurance } \\
\hline No & 37 & 5.8 & 30 & 6.7 & 7 & 3.6 \\
\hline Yes & 603 & 94.2 & 418 & 93.3 & 185 & 96.4 \\
\hline
\end{tabular}

TABLE 2: Baseline data of clinicopathological characteristics of patients with GCLM in CSS group.

\begin{tabular}{|c|c|c|c|c|c|c|}
\hline \multirow[t]{2}{*}{ Variables } & \multicolumn{2}{|c|}{ Total cohort $(N=524)$} & \multicolumn{2}{|c|}{$\begin{array}{l}\text { Training cohort } \\
\qquad(N=368)\end{array}$} & \multicolumn{2}{|c|}{$\begin{array}{c}\text { Validation cohort } \\
\quad(N=156)\end{array}$} \\
\hline & $n$ & $\%$ & $n$ & $\%$ & $n$ & $\%$ \\
\hline \multicolumn{7}{|l|}{ Age } \\
\hline$<60$ & 197 & 37.6 & 131 & 35.6 & 66 & 42.3 \\
\hline$\geq 60$ & 327 & 62.4 & 237 & 64.4 & 90 & 57.7 \\
\hline \multicolumn{7}{|l|}{ Race } \\
\hline Black & 67 & 12.8 & 50 & 13.6 & 17 & 10.9 \\
\hline Other & 69 & 13.2 & 49 & 13.3 & 20 & 12.8 \\
\hline White & 388 & 74 & 269 & 73.1 & 119 & 76.3 \\
\hline \multicolumn{7}{|l|}{ Sex } \\
\hline Female & 162 & 30.9 & 109 & 29.6 & 53 & 34.0 \\
\hline Male & 362 & 69.1 & 259 & 70.4 & 103 & 66.0 \\
\hline \multicolumn{7}{|l|}{ Histologic type } \\
\hline Adenocarcinoma & 340 & 64.9 & 247 & 67.1 & 93 & 59.6 \\
\hline Signet ring cell & 76 & 14.5 & 46 & 12.5 & 30 & 19.2 \\
\hline Intestinal type & 36 & 6.9 & 26 & 7.1 & 10 & 6.4 \\
\hline Other & 72 & 13.7 & 49 & 13.3 & 23 & 14.7 \\
\hline \multicolumn{7}{|l|}{ T stage } \\
\hline $\mathrm{T} 1$ & 209 & 39.9 & 148 & 40.2 & 61 & 39.1 \\
\hline $\mathrm{T} 2$ & 36 & 6.9 & 27 & 7.3 & 9 & 5.8 \\
\hline $\mathrm{T} 3$ & 117 & 22.3 & 84 & 22.8 & 33 & 21.2 \\
\hline $\mathrm{T} 4$ & 162 & 30.9 & 109 & 29.6 & 53 & 34.0 \\
\hline \multicolumn{7}{|l|}{$\mathrm{N}$ stage } \\
\hline No & 207 & 39.5 & 144 & 39.1 & 63 & 40.4 \\
\hline N1 & 254 & 48.5 & 187 & 50.8 & 67 & 42.9 \\
\hline N2 & 26 & 5 & 14 & 3.8 & 12 & 7.7 \\
\hline N3 & 37 & 7.1 & 23 & 6.3 & 14 & 9.0 \\
\hline \multicolumn{7}{|l|}{ Grade } \\
\hline Grade I & 19 & 3.6 & 15 & 4.1 & 4 & 2.6 \\
\hline Grade II & 147 & 28.1 & 105 & 28.5 & 42 & 26.9 \\
\hline Grade III & 349 & 66.6 & 242 & 65.8 & 107 & 68.6 \\
\hline Grade IV & 9 & 1.7 & 6 & 1.6 & 3 & 1.9 \\
\hline \multicolumn{7}{|l|}{ Bone metastasis } \\
\hline No & 432 & 97.1 & 303 & 82.3 & 129 & 82.7 \\
\hline Yes & 92 & 2.9 & 65 & 17.7 & 27 & 17.3 \\
\hline \multicolumn{7}{|l|}{ Liver metastasis } \\
\hline No & 257 & 49 & 179 & 48.6 & 78 & 50.0 \\
\hline Yes & 267 & 51 & 189 & 51.4 & 78 & 50.0 \\
\hline \multicolumn{7}{|l|}{ Brain metastasis } \\
\hline No & 509 & 97.1 & 356 & 96.7 & 153 & 98.1 \\
\hline Yes & 15 & 2.9 & 12 & 3.3 & 3 & 1.9 \\
\hline \multicolumn{7}{|l|}{ Primary site } \\
\hline Cardia & 240 & 45.8 & 164 & 44.6 & 76 & 48.7 \\
\hline Fundus & 33 & 6.3 & 22 & 6 & 11 & 7.1 \\
\hline Body & 38 & 7.3 & 29 & 7.9 & 9 & 5.8 \\
\hline Gastric antrum & 63 & 12 & 46 & 12.5 & 17 & 10.9 \\
\hline Lesser & 20 & 3.8 & 11 & 3 & 9 & 5.8 \\
\hline Greater & 24 & 4.6 & 19 & 5.2 & 5 & 3.2 \\
\hline Other & 106 & 20.2 & 77 & 20.9 & 29 & 18.6 \\
\hline
\end{tabular}


TABLE 2: Continued.

\begin{tabular}{|c|c|c|c|c|c|c|}
\hline \multirow[t]{2}{*}{ Variables } & \multicolumn{2}{|c|}{ Total cohort $(N=524)$} & \multicolumn{2}{|c|}{$\begin{array}{l}\text { Training cohort } \\
\qquad(N=368)\end{array}$} & \multicolumn{2}{|c|}{$\begin{array}{c}\text { Validation cohort } \\
\quad(N=156)\end{array}$} \\
\hline & $n$ & $\%$ & $n$ & $\%$ & $n$ & $\%$ \\
\hline \multicolumn{7}{|c|}{ Radiotherapy } \\
\hline No & 401 & 76.5 & 276 & 75 & 125 & 80.1 \\
\hline Yes & 123 & 23.5 & 92 & 25 & 31 & 19.9 \\
\hline \multicolumn{7}{|c|}{ Chemotherapy } \\
\hline No & 205 & 39.1 & 158 & 42.9 & 47 & 30.1 \\
\hline Yes & 319 & 60.9 & 210 & 57.1 & 109 & 69.9 \\
\hline \multicolumn{7}{|l|}{ Surgery } \\
\hline No & 481 & 91.8 & 340 & 92.4 & 141 & 90.4 \\
\hline Yes & 43 & 8.2 & 28 & 7.6 & 15 & 9.6 \\
\hline \multicolumn{7}{|c|}{ Marital status } \\
\hline No & 209 & 39.9 & 152 & 41.3 & 57 & 36.5 \\
\hline Yes & 315 & 60.1 & 216 & 58.7 & 99 & 63.5 \\
\hline \multicolumn{7}{|l|}{ Insurance } \\
\hline No & 31 & 5.9 & 22 & 6 & 9 & 5.8 \\
\hline Yes & 493 & 94.1 & 346 & 94 & 147 & 94.2 \\
\hline
\end{tabular}

TAвLe 3: Univariate and multivariate Cox proportional hazards regression analysis of patients with GCLM in the OS group.

\begin{tabular}{|c|c|c|c|c|}
\hline \multirow{2}{*}{ Variables } & \multicolumn{2}{|c|}{ Univariate Cox regression analysis } & \multicolumn{2}{|c|}{ Multivariate Cox regression analysis } \\
\hline & HR $(95 \% \mathrm{CI})$ & $P$ & HR $(95 \% \mathrm{CI})$ & $P$ \\
\hline \multicolumn{5}{|l|}{ Age } \\
\hline$<60$ & Reference & & & \\
\hline$\geq 60$ & $1.044(0.854-1.277)$ & 0.671 & & \\
\hline \multicolumn{5}{|l|}{ Race } \\
\hline Black & Reference & & & \\
\hline Other & $0.979(0.658-1.457)$ & 0.918 & & \\
\hline White & $0.788(0.586-1.061)$ & 0.117 & & \\
\hline \multicolumn{5}{|l|}{ Sex } \\
\hline Female & Reference & & & \\
\hline Male & $0.99(0.799-1.225)$ & 0.923 & & \\
\hline \multicolumn{5}{|l|}{ Histologic type } \\
\hline Adenocarcinoma & Reference & & & \\
\hline Signet ring cell & $1.044(0.786-1.388)$ & 0.765 & & \\
\hline Intestinal type & $1.358(0.901-2.047)$ & 0.144 & & \\
\hline Other & $1.144(0.873-1.498)$ & 0.33 & & \\
\hline \multicolumn{5}{|l|}{$\mathrm{T}$ stage } \\
\hline $\mathrm{T} 1$ & Reference & & & \\
\hline $\mathrm{T} 2$ & $0.705(0.459-1.083)$ & 0.11 & & \\
\hline $\mathrm{T} 3$ & $0.944(0.735-1.214)$ & 0.656 & & \\
\hline $\mathrm{T} 4$ & $1.219(0.969-1.535)$ & 0.091 & & \\
\hline \multicolumn{5}{|l|}{$\mathrm{N}$ stage } \\
\hline No & Reference & & & \\
\hline N1 & $1.009(0.820-1.243)$ & 0.929 & & \\
\hline N2 & $0.961(0.664-1.391)$ & 0.833 & & \\
\hline N3 & $1.166(0.790-1.721)$ & 0.44 & & \\
\hline \multicolumn{5}{|l|}{ Grade } \\
\hline Grade I & Reference & & Reference & \\
\hline Grade II & $1.458(0.850-2.501)$ & 0.171 & $1.275(0.738-2.201)$ & 0.383 \\
\hline Grade III & $1.864(1.105-3.144)$ & 0.02 & $1.896(1.118-3.214)$ & 0.018 \\
\hline Grade IV & $1.238(0.410-3.743)$ & 0.705 & $0.942(0.310-2.864)$ & 0.916 \\
\hline \multicolumn{5}{|l|}{ Bone metastasis } \\
\hline No & Reference & & & \\
\hline Yes & $1.075(0.833-1.388)$ & 0.58 & & \\
\hline \multicolumn{5}{|l|}{ Liver metastasis } \\
\hline No & Reference & & & \\
\hline Yes & $1.309(1.080-1.587)$ & 0.006 & $1.440(1.179-1.760)$ & $<0.001$ \\
\hline
\end{tabular}


TABLE 3: Continued.

\begin{tabular}{|c|c|c|c|c|}
\hline \multirow{2}{*}{ Variables } & \multicolumn{2}{|c|}{ Univariate Cox regression analysis } & \multicolumn{2}{|c|}{ Multivariate Cox regression analysis } \\
\hline & HR $(95 \% \mathrm{CI})$ & $P$ & $\mathrm{HR}(95 \% \mathrm{CI})$ & $P$ \\
\hline \multicolumn{5}{|l|}{ Brain metastasis } \\
\hline No & Reference & & & \\
\hline Yes & $1.434(0.806-2.550)$ & 0.22 & & \\
\hline \multicolumn{5}{|l|}{ Primary site } \\
\hline Cardia & Reference & & & \\
\hline Fundus & $1.487(0.956-2.315)$ & 0.079 & & \\
\hline Body & $1.179(0.792-1.754)$ & 0.418 & & \\
\hline Gastric antrum & $1.224(0.893-1.676)$ & 0.208 & & \\
\hline Lesser & $1.211(0.764-1.922)$ & 0.416 & & \\
\hline Greater & $1.182(0.737-1.895)$ & 0.488 & & \\
\hline Other & $1.28(0.995-1.646)$ & 0.054 & & \\
\hline \multicolumn{5}{|l|}{ Radiotherapy } \\
\hline No & Reference & & & \\
\hline Yes & $0.761(0.606-0.955)$ & 0.019 & $0.979(0.770-1.244)$ & 0.859 \\
\hline \multicolumn{5}{|l|}{ Chemotherapy } \\
\hline No & Reference & & & \\
\hline Yes & $0.312(0.253-0.384)$ & $<0.001$ & $0.292(0.235-0.363)$ & $<0.001$ \\
\hline \multicolumn{5}{|l|}{ Surgery } \\
\hline No & Reference & & & \\
\hline Yes & $0.835(0.598-1.167)$ & 0.291 & & \\
\hline \multicolumn{5}{|l|}{ Marital status } \\
\hline No & Reference & & & \\
\hline Yes & $0.844(0.694-1.025)$ & 0.087 & & \\
\hline \multicolumn{5}{|l|}{ Insurance } \\
\hline No & Reference & & & \\
\hline Yes & $1.076(0.723-1.602)$ & 0.718 & & \\
\hline
\end{tabular}

TABLE 4: Univariate and multivariate Cox proportional hazards regression analysis of patients with GCLM in the CSS group.

\begin{tabular}{|c|c|c|c|c|}
\hline \multirow{2}{*}{ Variables } & \multicolumn{2}{|c|}{ Univariate Cox regression analysis } & \multicolumn{2}{|c|}{ Multivariate Cox regression analysis } \\
\hline & $\operatorname{HR}(95 \% \mathrm{CI})$ & $P$ & $\operatorname{HR}(95 \% \mathrm{CI})$ & $P$ \\
\hline \multicolumn{5}{|l|}{ Age } \\
\hline$<60$ & Reference & & & \\
\hline$\geq 60$ & $0.945(0.759-1.176)$ & 0.612 & & \\
\hline \multicolumn{5}{|l|}{ Race } \\
\hline Black & Reference & & Reference & \\
\hline Other & $0.823(0.549-1.232)$ & 0.343 & $0.916(0.602-1.393)$ & 0.681 \\
\hline White & $0.707(0.519-0.963)$ & 0.028 & $0.883(0.635-1.227)$ & 0.458 \\
\hline \multicolumn{5}{|l|}{ Sex } \\
\hline Female & Reference & & & \\
\hline Male & $0.976(0.775-1.230)$ & 0.837 & & \\
\hline \multicolumn{5}{|l|}{ Histologic type } \\
\hline Adenocarcinoma & Reference & & & \\
\hline Signet ring cell & $1.064(0.763-1.484)$ & 0.715 & & \\
\hline Intestinal type & $1.207(0.798-1.825)$ & 0.372 & & \\
\hline Other & $1.152(0.846-1.570)$ & 0.368 & & \\
\hline \multicolumn{5}{|l|}{ T stage } \\
\hline $\mathrm{T} 1$ & Reference & & Reference & \\
\hline $\mathrm{T} 2$ & $0.594(0.378-0.932)$ & 0.024 & $0.71(0.446-1.131)$ & 0.149 \\
\hline $\mathrm{T} 3$ & $0.854(0.648-1.124)$ & 0.26 & $1.121(0.840-1.497)$ & 0.436 \\
\hline $\mathrm{T} 4$ & $1.088(0.844-1.402)$ & 0.513 & $1.15(0.882-1.498)$ & 0.302 \\
\hline \multicolumn{5}{|l|}{$\mathrm{N}$ stage } \\
\hline No & Reference & & & \\
\hline N1 & $0.922(0.737-1.153)$ & 0.475 & & \\
\hline $\mathrm{N} 2$ & $0.864(0.498-1.500)$ & 0.603 & & \\
\hline N3 & $0.865(0.551-1.358)$ & 0.528 & & \\
\hline
\end{tabular}


TABLE 4: Continued.

\begin{tabular}{|c|c|c|c|c|}
\hline \multirow{2}{*}{ Variables } & \multicolumn{2}{|c|}{ Univariate Cox regression analysis } & \multicolumn{2}{|c|}{ Multivariate Cox regression analysis } \\
\hline & $\operatorname{HR}(95 \% \mathrm{CI})$ & $P$ & $\operatorname{HR}(95 \% \mathrm{CI})$ & $P$ \\
\hline \multicolumn{5}{|l|}{ Grade } \\
\hline Grade I & Reference & & & \\
\hline Grade II & $1.282(0.732-2.245)$ & 0.385 & & \\
\hline Grade III & $1.632(0.950-2.806)$ & 0.076 & & \\
\hline Grade IV & $2.121(0.813-5.530)$ & 0.124 & & \\
\hline \multicolumn{5}{|l|}{ Bone metastasis } \\
\hline No & Reference & & & \\
\hline Yes & & 0.146 & & \\
\hline \multicolumn{5}{|l|}{ Liver metastasis } \\
\hline No & Reference & & Reference & \\
\hline Yes & $1.47(1.186-1.821)$ & $<0.001$ & $1.524(1.217-1.909)$ & $<0.001$ \\
\hline \multicolumn{5}{|l|}{ Brain metastasis } \\
\hline No & Reference & & & \\
\hline Yes & $1.114(0.610-2.033)$ & 0.725 & & \\
\hline \multicolumn{5}{|l|}{ Primary site } \\
\hline Cardia & Reference & & Reference & \\
\hline Fundus & $1.593(1.006-2.522)$ & 0.047 & $1.312(0.824-2.091)$ & 0.253 \\
\hline Body & $1.337(0.880-2.030)$ & 0.173 & $1.364(0.882-2.108)$ & 0.163 \\
\hline Gastric antrum & $1.498(1.067-2.105)$ & 0.02 & $1.206(0.846-1.718)$ & 0.301 \\
\hline Lesser & $1.185(0.642-2.189)$ & 0.587 & $1.409(0.732-2.710)$ & 0.305 \\
\hline Greater & $2.024(1.238-3.308)$ & 0.005 & $2.315(1.395-3.841)$ & 0.001 \\
\hline Other & $1.367(1.035-1.805)$ & 0.028 & $1.26(0.932-1.704)$ & 0.133 \\
\hline \multicolumn{5}{|l|}{ Radiotherapy } \\
\hline No & Reference & & & \\
\hline Yes & $0.813(0.640-1.033)$ & 0.091 & & \\
\hline \multicolumn{5}{|l|}{ Chemotherapy } \\
\hline No & Reference & & Reference & \\
\hline Yes & $0.388(0.311-0.484)$ & $<0.001$ & $0.398(0.317-0.501)$ & $<0.001$ \\
\hline \multicolumn{5}{|l|}{ Surgery } \\
\hline No & Reference & & & \\
\hline Yes & $0.715(0.474-1.077)$ & 0.109 & & \\
\hline \multicolumn{5}{|l|}{ Marital status } \\
\hline No & Reference & & Reference & \\
\hline Yes & $0.735(0.592-0.912)$ & 0.005 & $0.788(0.629-0.988)$ & 0.039 \\
\hline \multicolumn{5}{|l|}{ Insurance } \\
\hline No & Reference & & & \\
\hline Yes & $0.954(0.600-1.518)$ & 0.843 & & \\
\hline
\end{tabular}

nomograms to the actual results (Figures 5(a)-5(f)). The calibration curves for the CSS probabilities at 3 , 6 , and 12 months also suggested the same better consistency among the CSS forecasted with the nomogram and the actual results (Figures 6(a)6(f)).

(iv) DCA curves: DCA curves confirmed that nomograms can better predict OS (Figures 7(a)-7(f)) and CSS (Figures 8(a)-8(f)) in patients with GCLM. In addition, K-M survival curves revealed that, for OS (Figures 9(a) and 9(b)) and CSS (Figures 9(c) and $9(\mathrm{~d})$ ), patients from the higher risk group had a more unfavorable prognosis than those from the lower risk group.

3.6. Establishment of Two Web-Based Nomograms. Based on the above results, we constructed a probabilistic calculator OS (https://zhenghh.shinyapps.io/aclmos/) and CSS (https://zhenghh.shinyapps.io/aslmcss/) based on a dynamic network, which predicts the OS and CSS of patients with GCLM based on previous nomograms (Figure 10(a)). For example, the CSS of a patient with GCLM, who is a married woman with liver metastases, occurs in the gastric body and without chemotherapy. The survival curve of this patient is shown in Figure 10(b). Survival rates and 95\% confidence intervals at three months (Figure 10(c), black line), six months (Figure 10(c), blue line), and 12 months (Figure 10(c), red line) can also be observed at the operation interface. In addition, specific numbers are summarized to improve the prediction accuracy (Figure 10(d)). The OS of patients with GCLM can be predicted in the same way.

\section{Discussion}

GC is a malignant tumor of the gastrointestinal tract with a low early diagnosis rate, low surgical resection rate, and high mortality rate [23]. The majority of patients with GC are in 


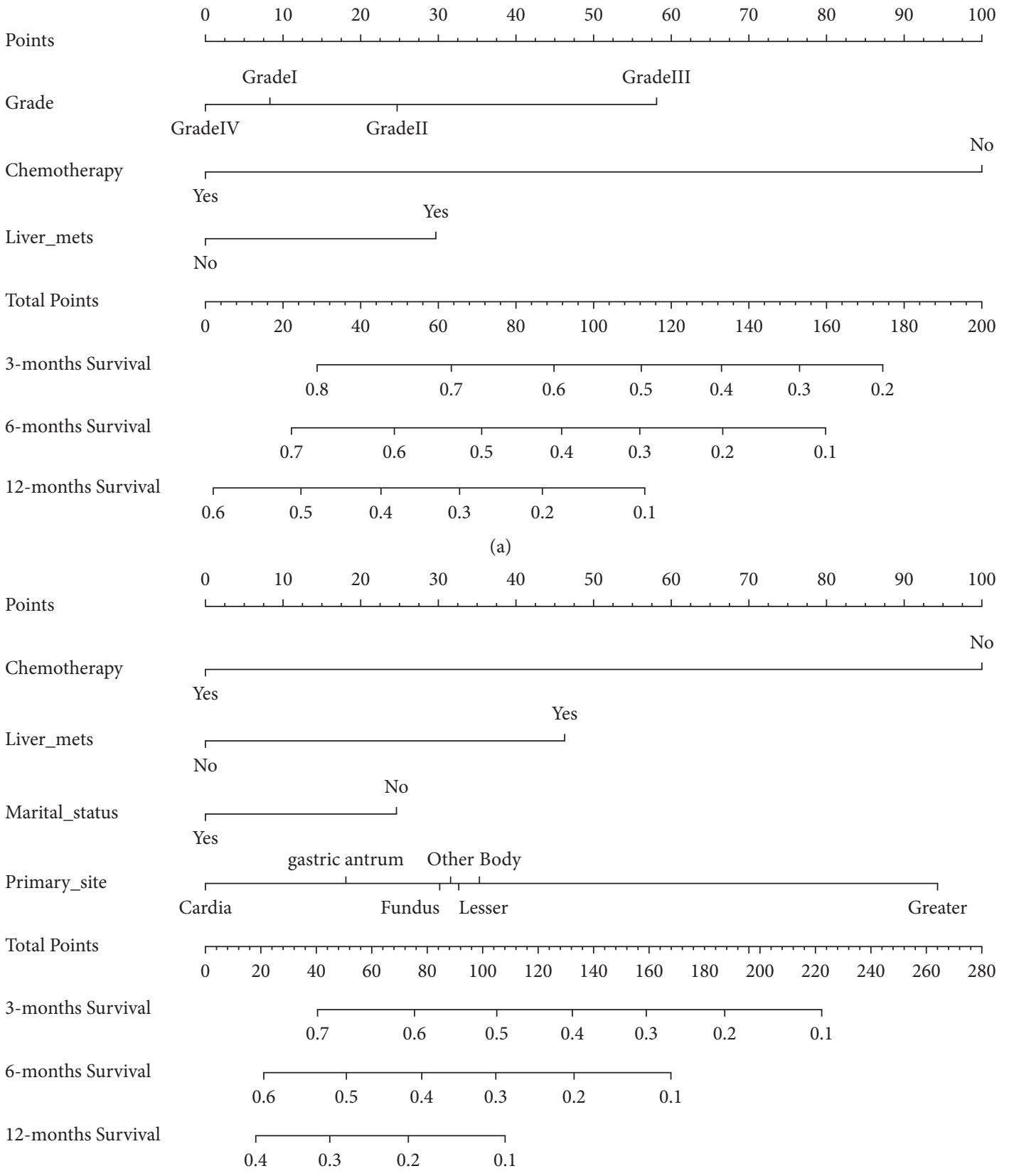

(b)

FIGURE 2: Nomogram. (a) Overall survival (OS) nomogram; (b) cancer-specific survival (CSS) nomogram. OS, overall survival; CSS, cancerspecific survival.

the advanced stage at the time of consultation, and 32.6\% have distant metastases [24]. Interestingly, the incidence of LM is $14.9 \%$ [24]. LM typically indicates advanced tumors, and when not detected and treated in time, the prognosis is extremely poor. In our study, we created two nomograms to predict the prognosis of patients with GCLM. These two nomograms performed well in predicting OS and CSS in patients with GCLM, allowing more precise individualized clinical decision-making and surveillance. Finally, we built two web-based nomograms based on the nomograms. This prediction model can facilitate the prediction of the survival probability of patients with GCLM at a specific time.
Clinicians can also arrange personalized treatment plans based on the prediction results.

As we know, survival statistics of GCLM are not optimistic. Therefore, clinicians can identify the risk and protective factors of GCLM, which can result in a good prognosis for patients with GCLM. A number of potential biomarkers that are involved in cadherin-catenin interaction, integrin signaling, and cancer stem cell identification in gastrointestinal cancers have been observed [25]. However, these biomarkers are difficult to measure, have low sensitivity, are expensive, and have few clinical applications. Therefore, it is necessary to actively identify other clinical 


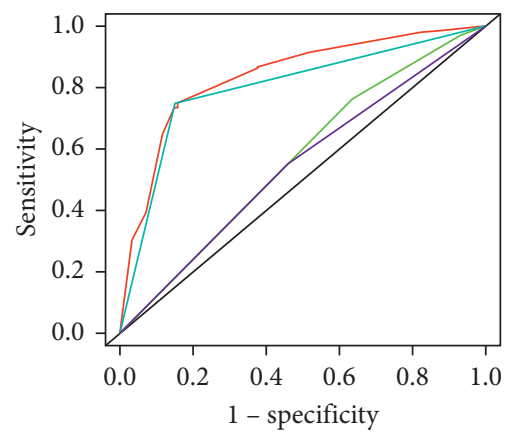

— Nomogram, AUC $=0.837$

- Grade, $\mathrm{AUC}=0.565$

Chemotherapy, AUC $=0.799$

— Liver_mets, AUC $=0.544$

(a)

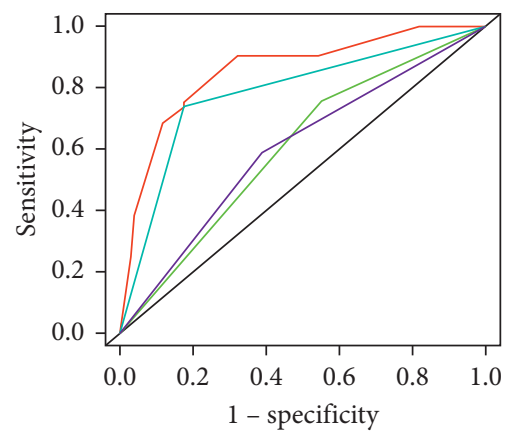

- Nomogram, AUC $=0.855$

- Grade, AUC $=0.601$

Chemotherapy, $\mathrm{AUC}=0.782$

— Liver_mets, AUC $=0.599$

(d)

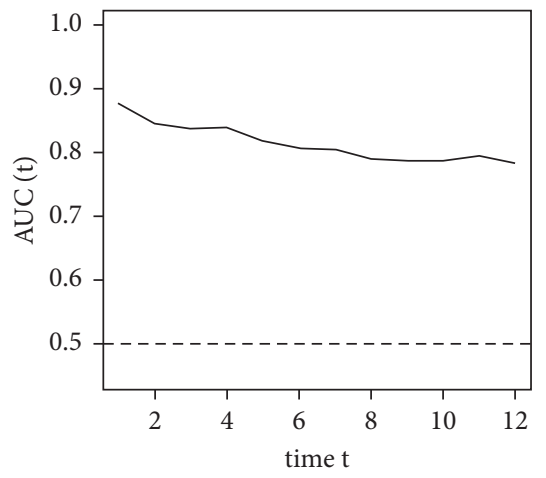

(g)

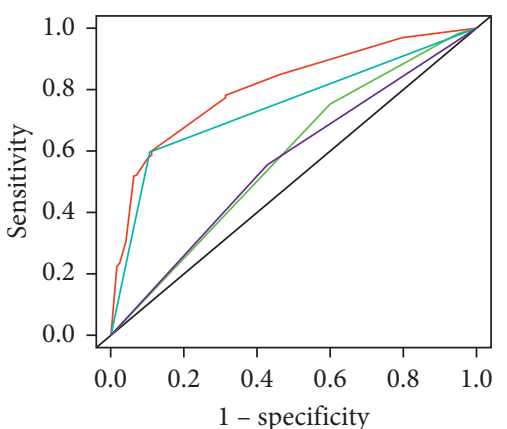

— Nomogram, AUC $=0.807$

- Grade, $\mathrm{AUC}=0.579$

Chemotherapy, AUC $=0.745$

— Liver_mets, AUC $=0.563$

(b)

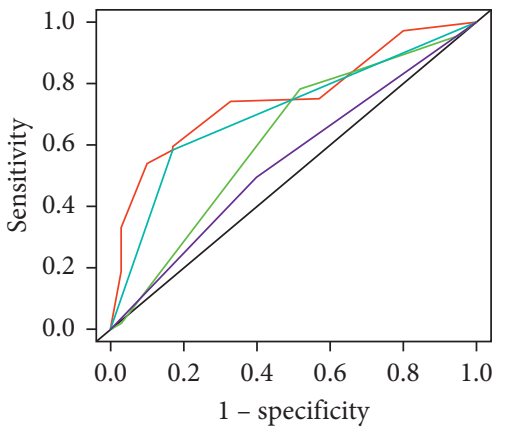

- Nomogram, AUC $=0.755$

Grade, AUC $=0.621$

- Chemotherapy, $\mathrm{AUC}=0.708$

_ Liver_mets, AUC $=0.548$

(e)

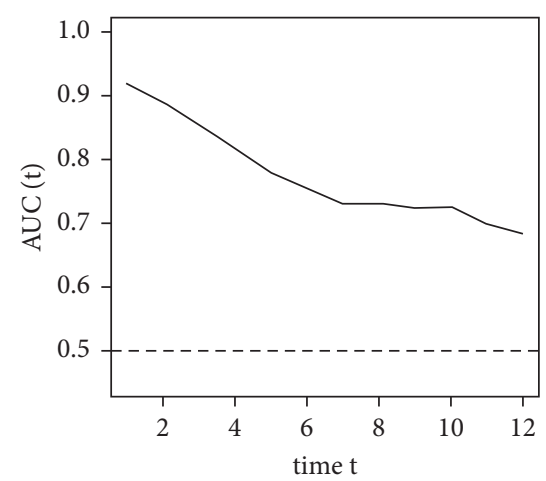

(h)

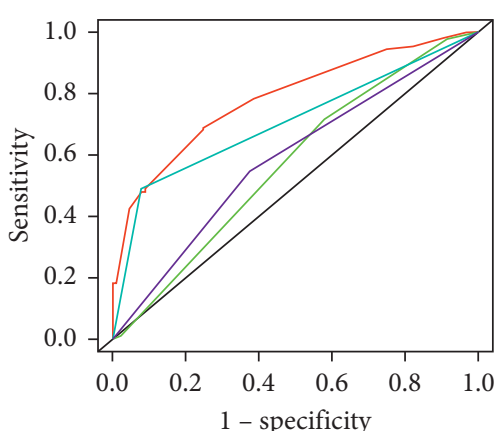

— Nomogram, AUC $=0.784$

- Grade, AUC $=0.570$

Chemotherapy, AUC $=0.706$

— Liver_mets, AUC $=0.585$

(c)
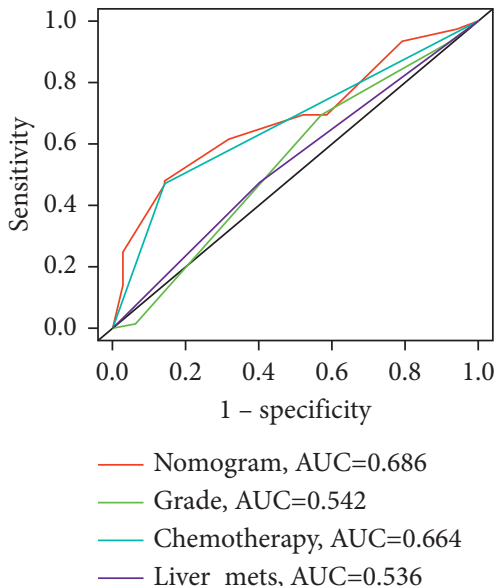

(f)

FIGURE 3: Receiver operating characteristic (ROC) curves of OS. (a-c) ROC curves corresponding to 3, 6, and 12months in the training cohort, respectively; (d-f) ROC curves corresponding to 3, 6, and 12 months in the verification cohort, respectively; (g) the time-dependent ROC curve corresponding to 1 to 12 months in the verification cohort in the training cohort; $3 \mathrm{~h}$, the time-dependent ROC curve corresponding to 1 to 12 months in the verification cohort. ROC, receiver operating characteristic; OS, overall survival.

features related to prognosis in patients with advanced GCLM. In 2019, Wenjie et al. [26] found that age, race, primary site, $\mathrm{T}$ stage, and $\mathrm{N}$ stage are independently related to CSS in patients with lymph node-positive GC. Studies have shown that the fat content in high muscle tissue is associated with CSS in patients with locally advanced GC [27]. However, so far, few studies have focused on GCLM, and no corresponding nomogram has been established to assess the survival and prognosis of these patients. Previous studies have confirmed that the prognostic factors of liver cancer are quite different from those of liver cancer with bone metastasis [28-31]. 


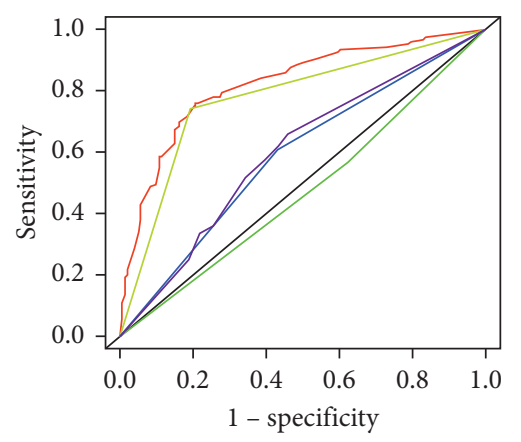

— Nomogram, AUC $=0.820$

Chemotherapy, AUC $=0.774$

—_Marital_status, AUC $=0.471$

— Liver_mets, AUC $=0.588$

— Primary_site, AUC $=0.599$

(a)

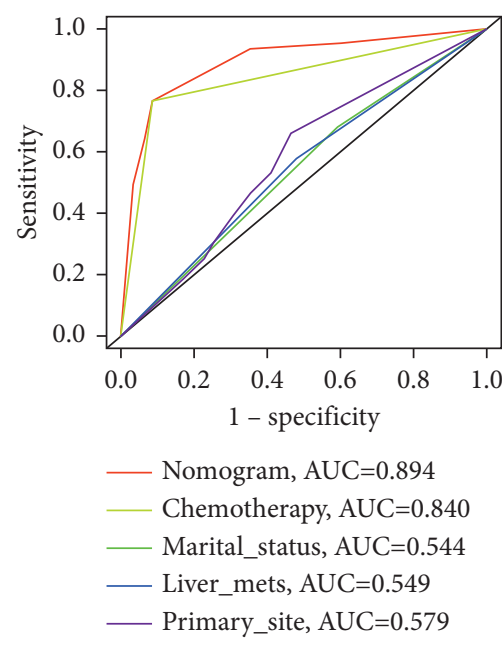

(d)

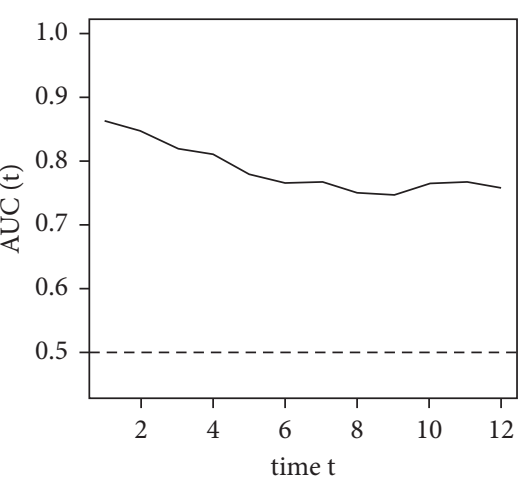

(g)

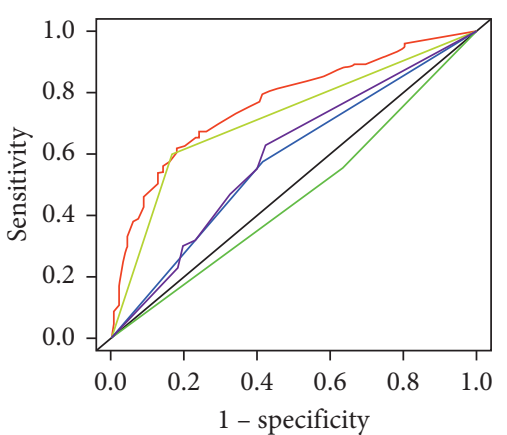

— Nomogram, AUC $=0.766$

Chemotherapy, AUC $=0.715$

Marital_status, AUC $=0.460$

— Liver_mets, AUC $=0.579$

— Primary_site, AUC $=0.594$

(b)

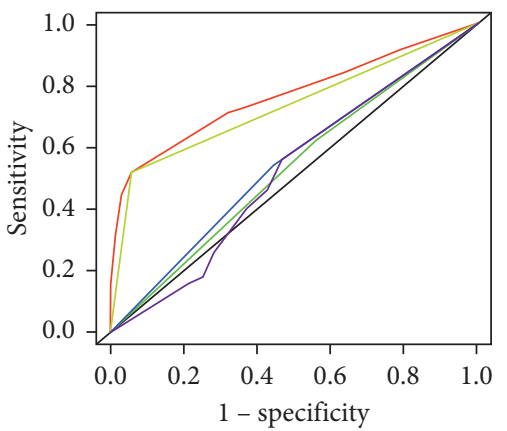

- Nomogram, AUC $=0.764$

Chemotherapy, $\mathrm{AUC}=0.730$

— Marital_status, AUC $=0.532$

— Liver_mets, AUC $=0.547$

— Primary_site, AUC $=0.519$

(e)

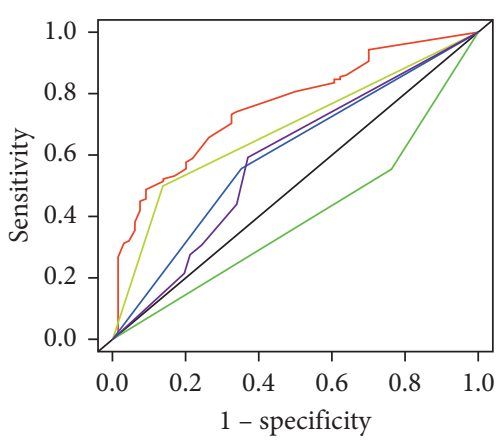

— Nomogram, AUC $=0.760$

Chemotherapy, AUC $=0.681$

- Marital_status, AUC $=0.394$

— Liver_mets, AUC $=0.602$

— Primary_site, AUC $=0.587$

(c)

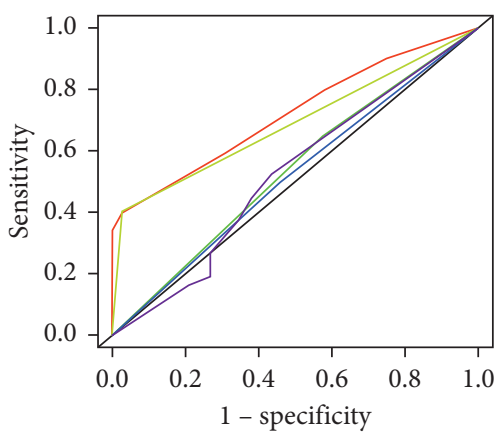

- Nomogram, AUC $=0.720$

Chemotherapy, $\mathrm{AUC}=0.689$

— Marital_status, AUC $=0.536$

— Liver_mets, AUC $=0.519$

— Primary_site, AUC $=0.524$

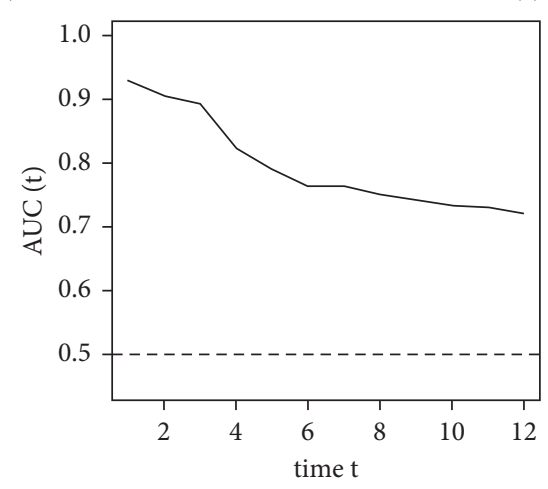

(h)

FIGURE 4: Receiver operating characteristic (ROC) curves of cancer-specific survival (CSS). (a-c) ROC curves corresponding to 3, 6, and 12 months in the training cohort, respectively; (d-f) ROC curves corresponding to 3, 6, and 12 months in the verification cohort, respectively; (g) the time-dependent ROC curve corresponding to 1 to 12 months in the verification cohort in the training cohort; (h) the time-dependent ROC curve corresponding to 1 to 12 months in the verification cohort. ROC, receiver operating characteristic; CSS, cancer-specific survival.

Therefore, it is not possible to evaluate the survival of patients with GCLM solely through the prognostic factors of GC, due to possible biases and errors. In this study, we screened the relevant independent prognostic factors of patients with GCLM. More meaningfully, this study integrates these multiple prognostic factors and visual graphs to predict the survival of patients with GCLM through nomograms, which is a practical tool widely used in oncology [32]. The web-based nomograms were based on further upgraded results. 


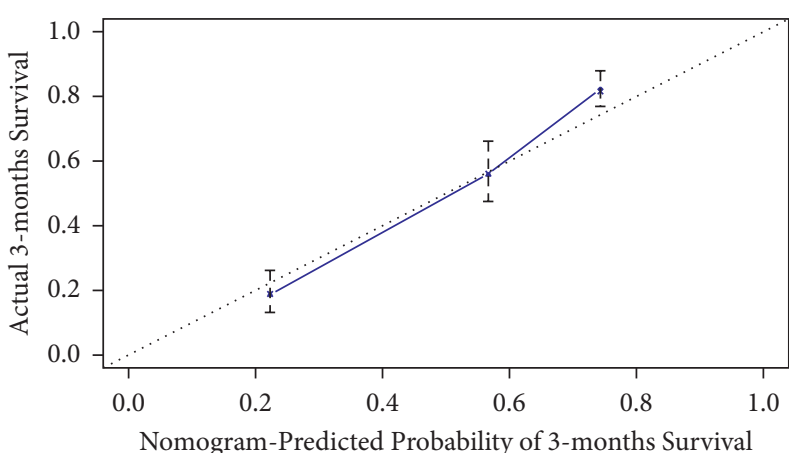

(a)

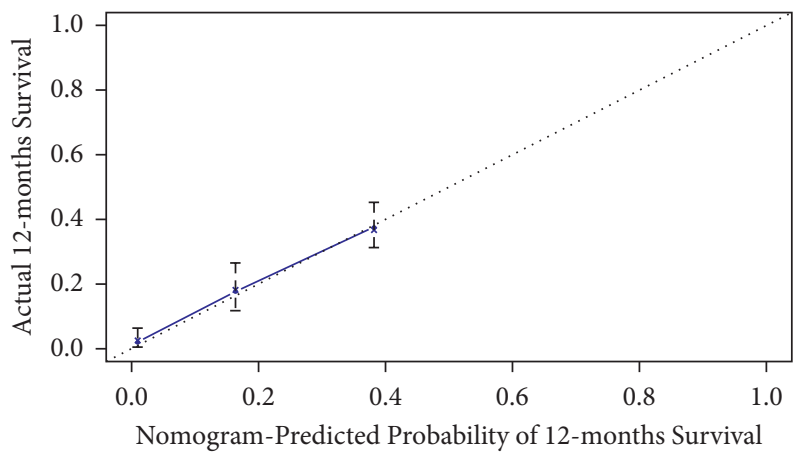

(c)

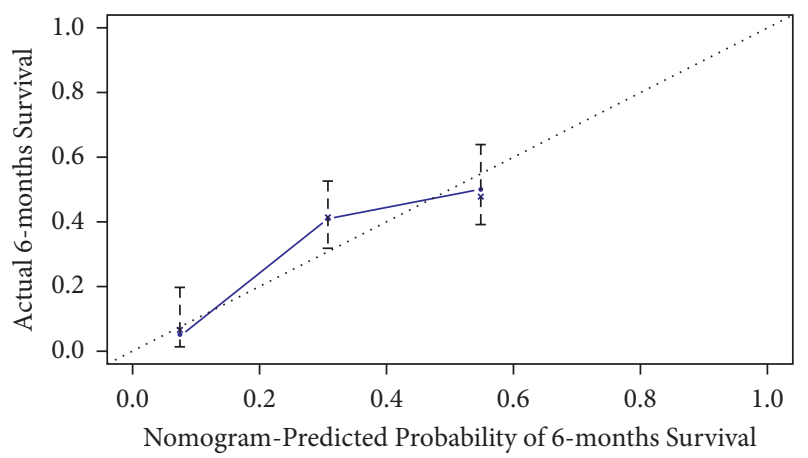

(e)

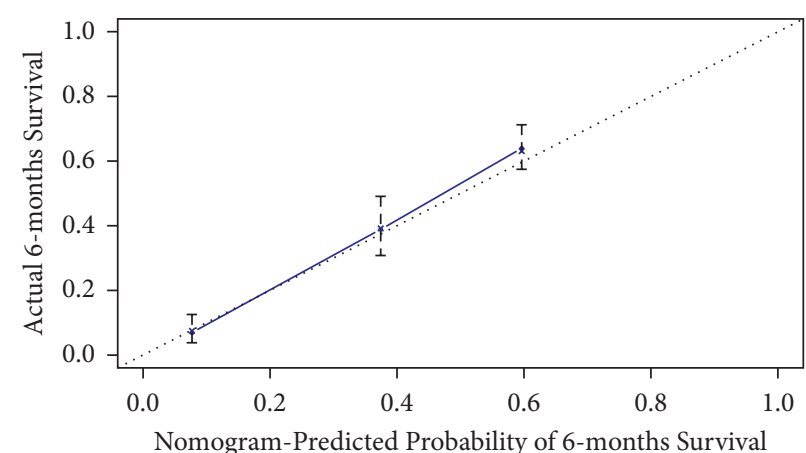

(b)

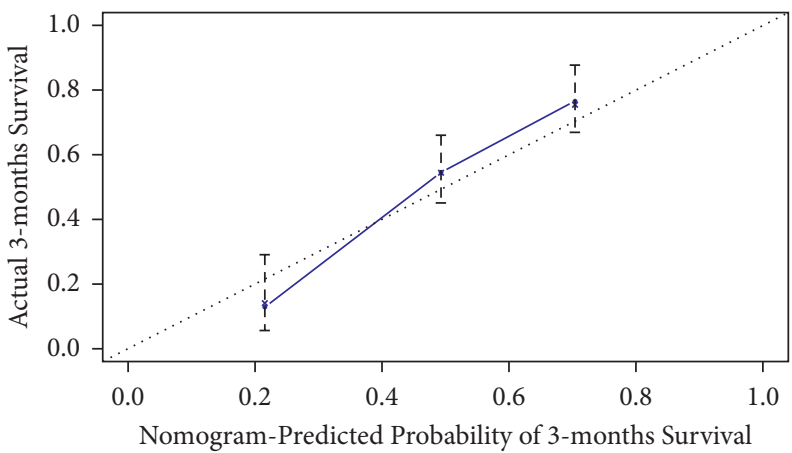

(d)

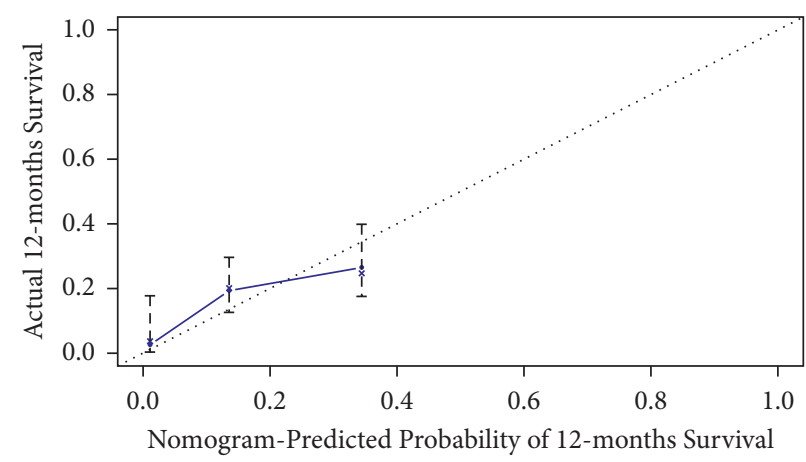

(f)

Figure 5: Calibration curves of overall survival (OS). (a-c) Calibration curves corresponding to 3, 6, and 12 months in the training cohort, respectively; (d-f) calibration curves corresponding to 3, 6, and 12 months in the verification cohort, respectively. OS, overall survival.

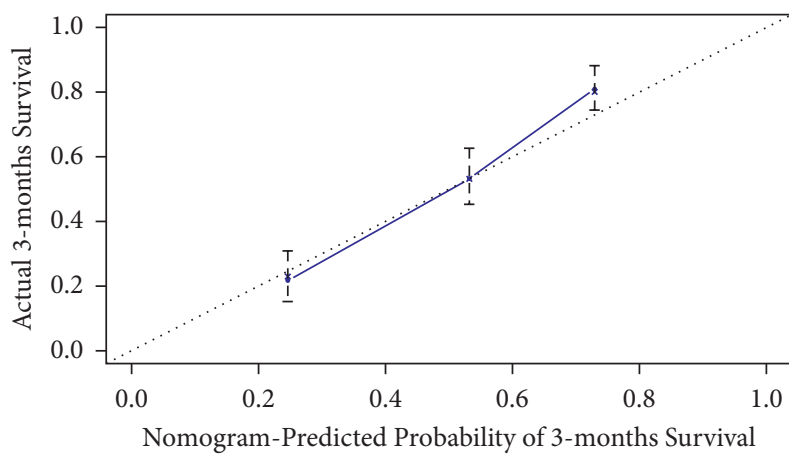

(a)

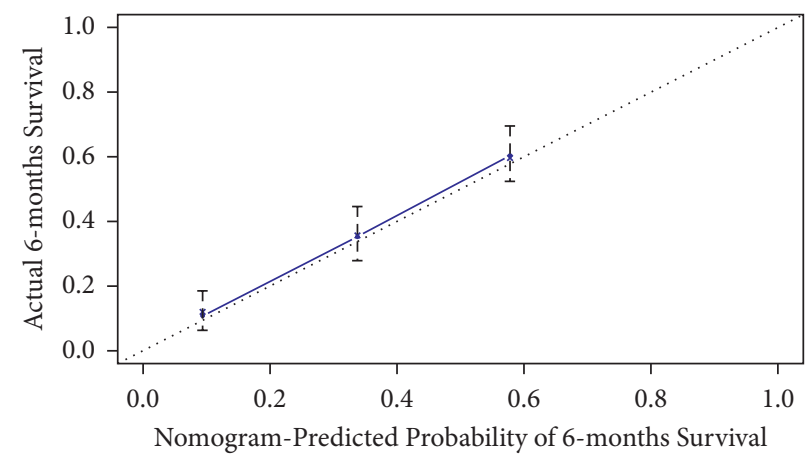

(b)

Figure 6: Continued. 


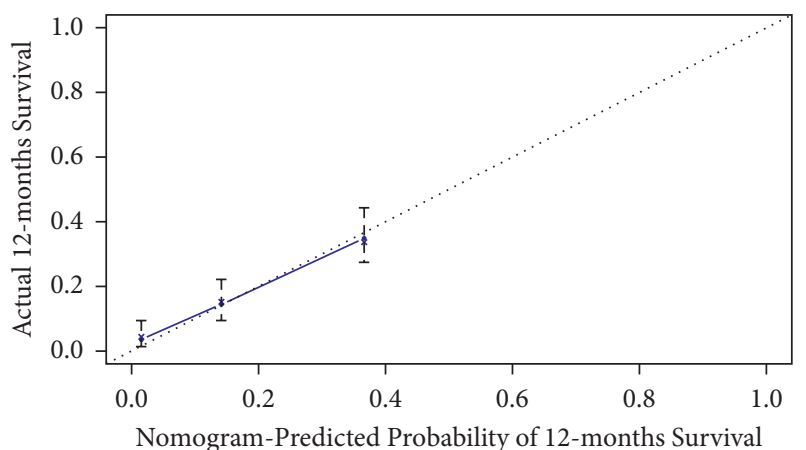

(c)

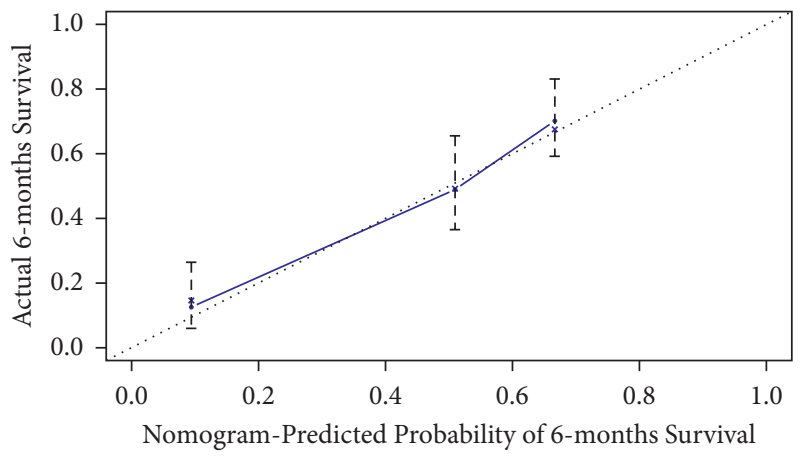

(e)

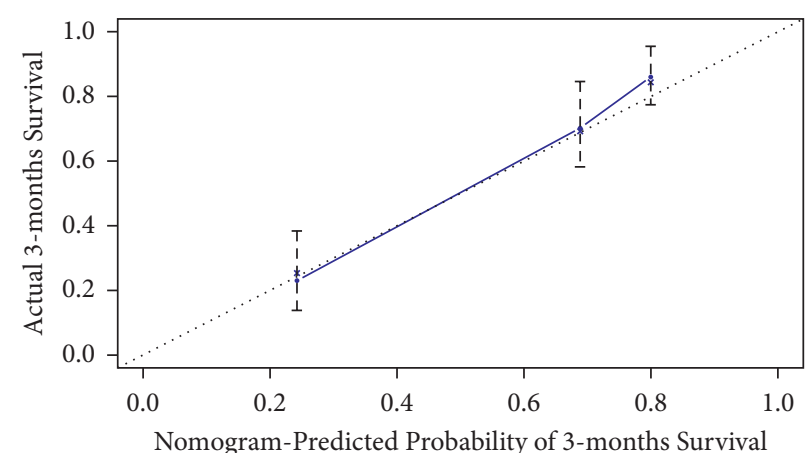

(d)

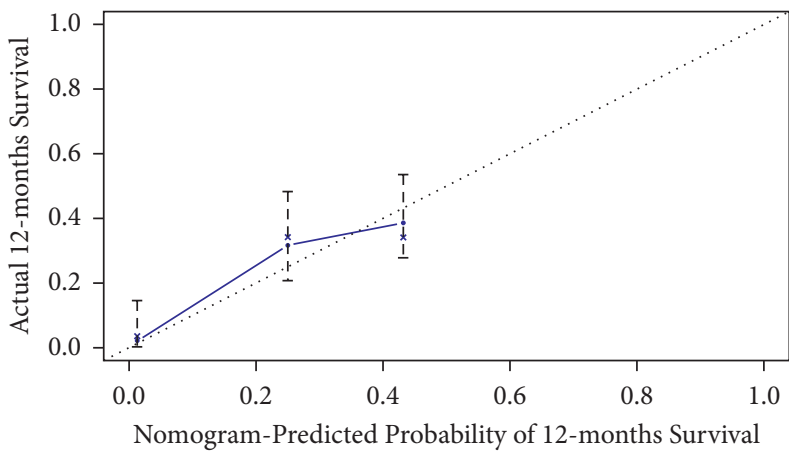

(f)

Figure 6: Calibration curves of cancer-specific survival (CSS). (a-c) Calibration curves corresponding to 3, 6, and 12 months in the training cohort, respectively; (d-f) calibration curves corresponding to 3, 6, and 12 months in the verification cohort, respectively. CSS, cancerspecific survival.

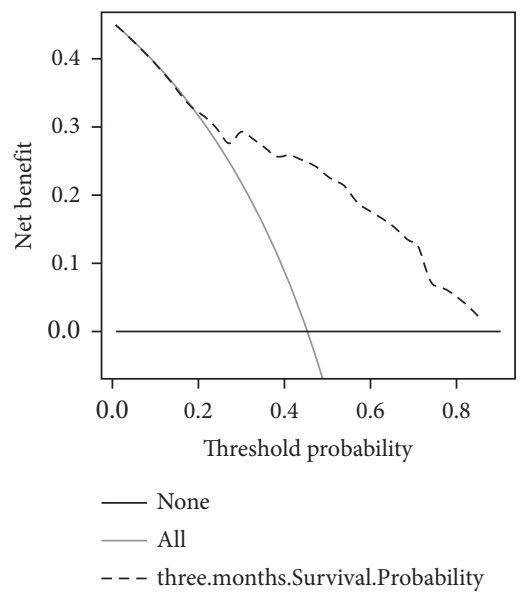

(a)

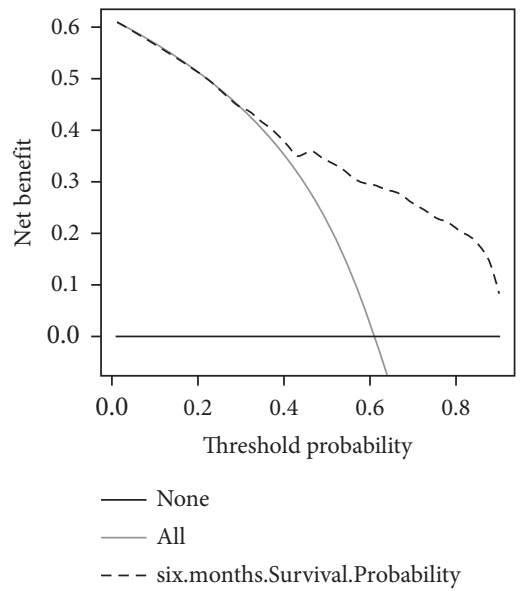

(b)

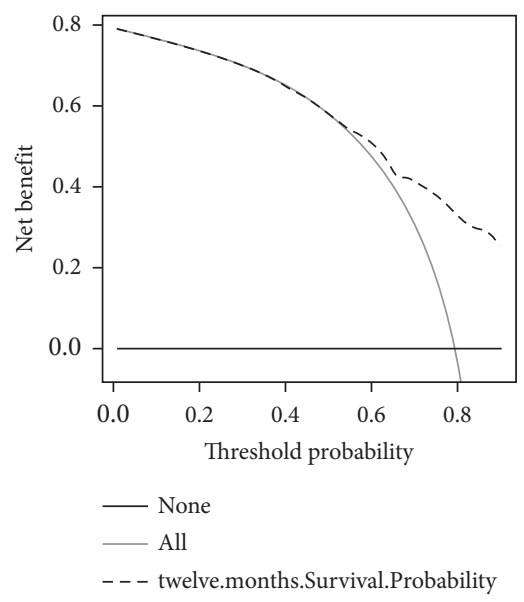

(c)

Figure 7: Continued. 


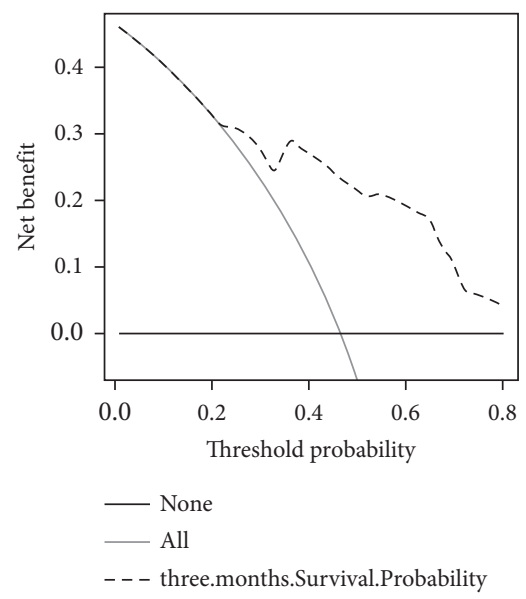

(d)

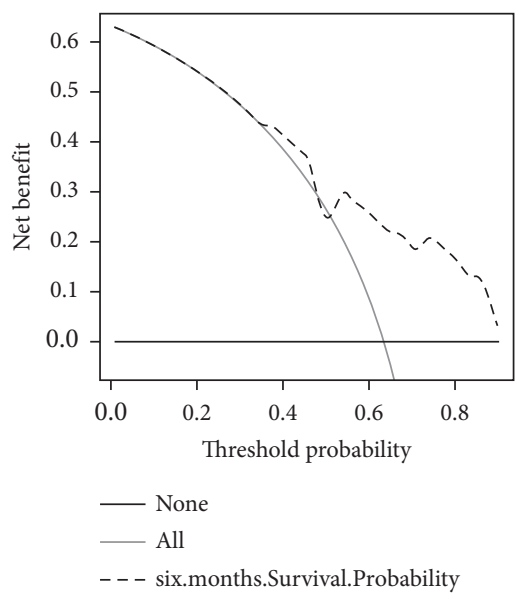

(e)

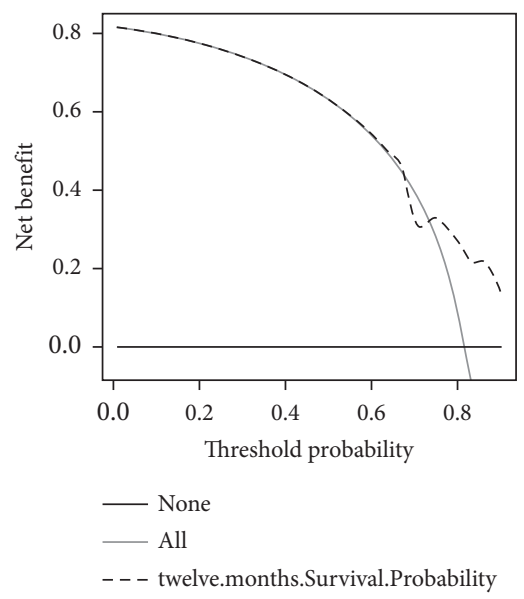

(f)

Figure 7: Decision curve analysis (DCA) curves of overall survival (OS). (a-c) DCA corresponding to 3, 6, and 12 months in the training cohort, respectively; (d-f) DCA corresponding to 3, 6, and 12 months in the verification cohort, respectively. DCA, decision curve analysis; OS, overall survival.

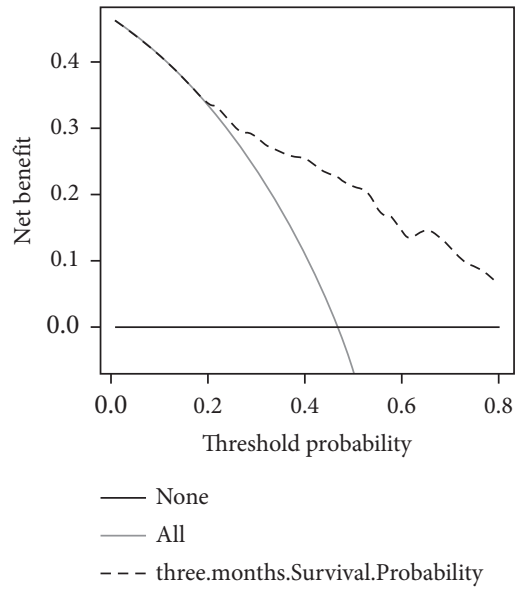

(a)

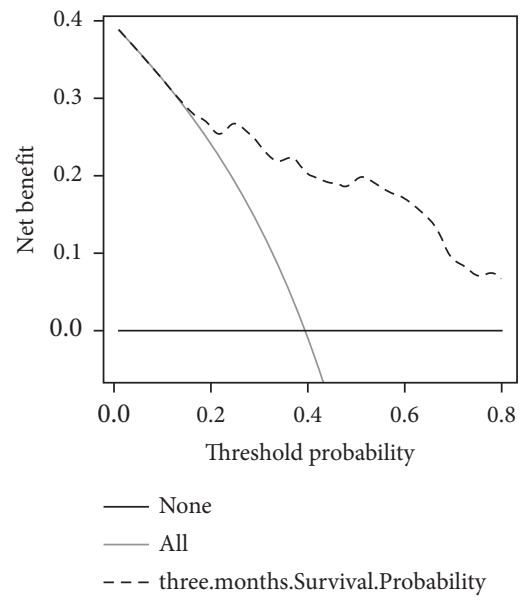

(d)

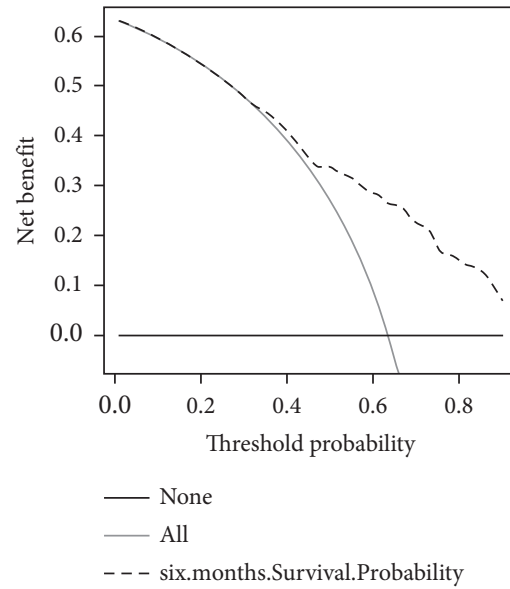

(b)

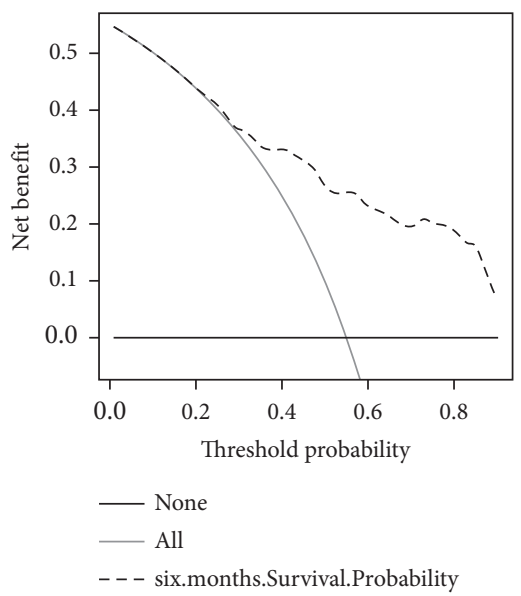

(e)

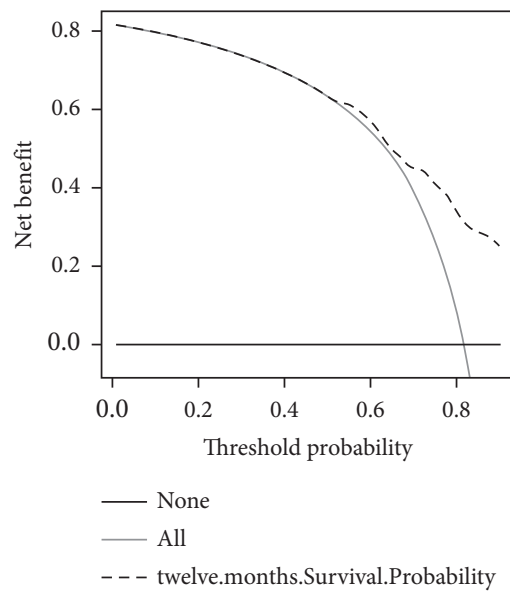

(c)

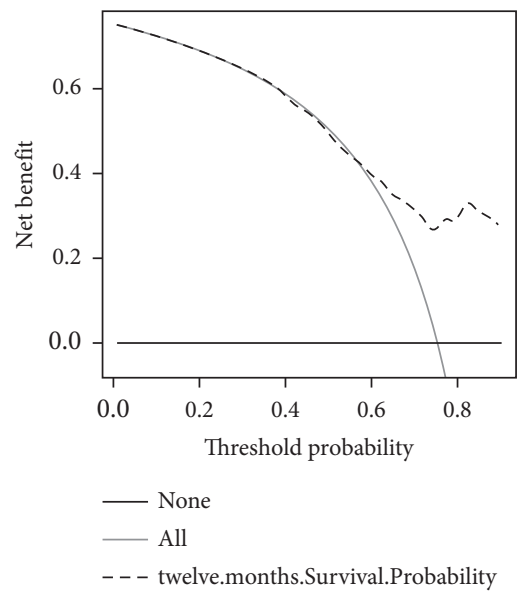

(f)

Figure 8: Decision curve analysis (DCA) curves of cancer-specific survival (CSS). (a-c) DCA corresponding to 3, 6, and 12 months in the training cohort, respectively; (d-f) DCA corresponding to 3, 6, and 12 months in the verification cohort, respectively. DCA, decision curve analysis; CSS, cancer-specific survival. 


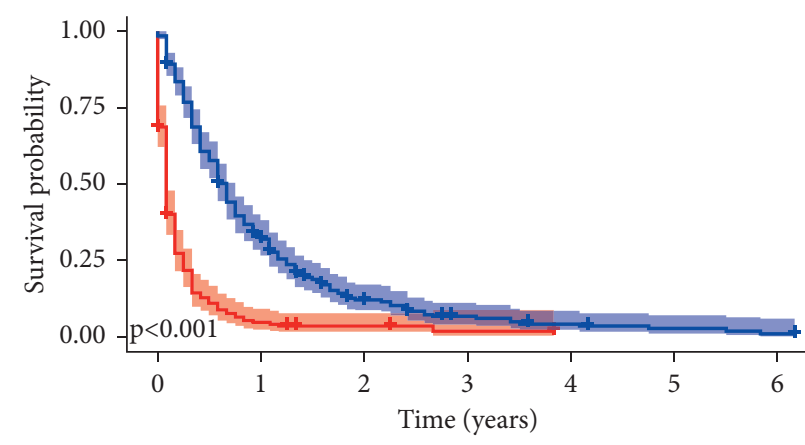

risk_score

+ High

+ Low

(a)

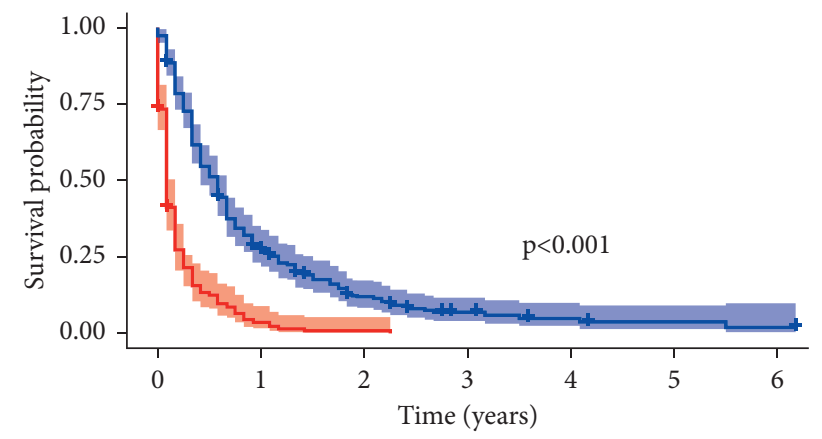

risk_score

+ High

+ Low

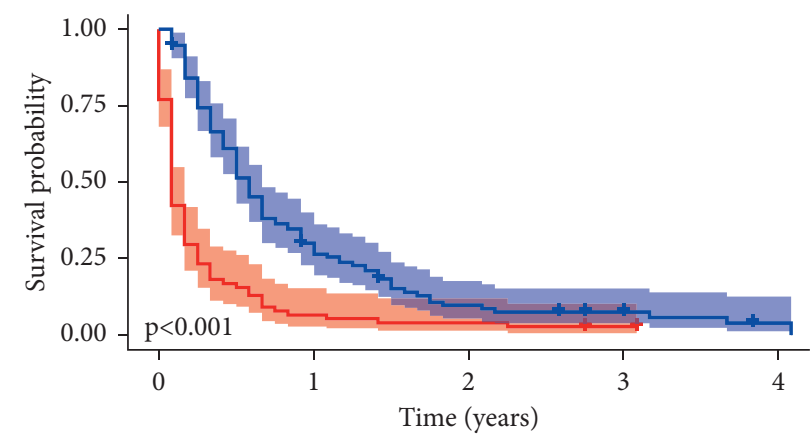

risk_score

++ High

+ Low

(b)

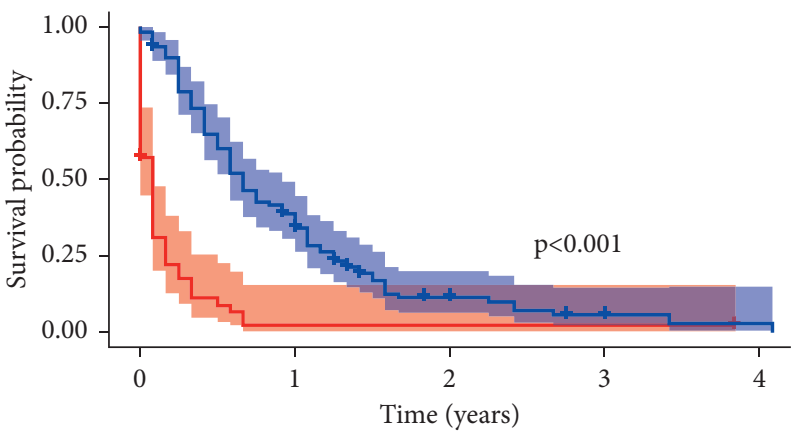

risk_score

+ High

++ Low

(c)

(d)

Figure 9: Kaplan-Meier (K-M) survival curves. (a) K-M survival curves in training cohort for OS of GCLM; (b) K-M survival curves in verification queue for OS of GCLM; (c) K-M survival curves in training cohort for CSS of GCLM; (d) K-M survival curves in verification cohort for CSS of GCLM. K-M, Kaplan-Meier; OS, overall survival; CSS, cancer-specific survival.

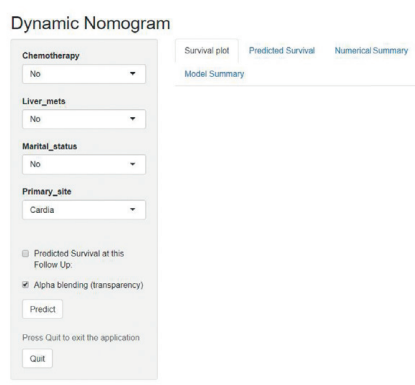

(a)

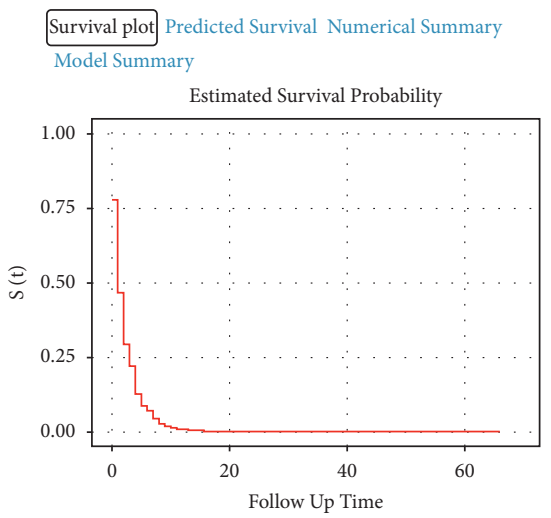

(b)

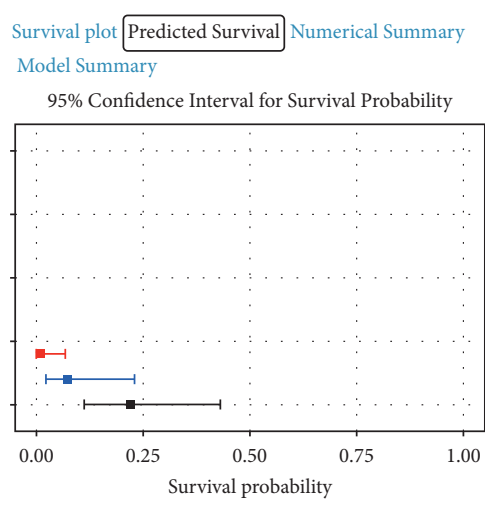

(c)

Figure 10: Continued. 


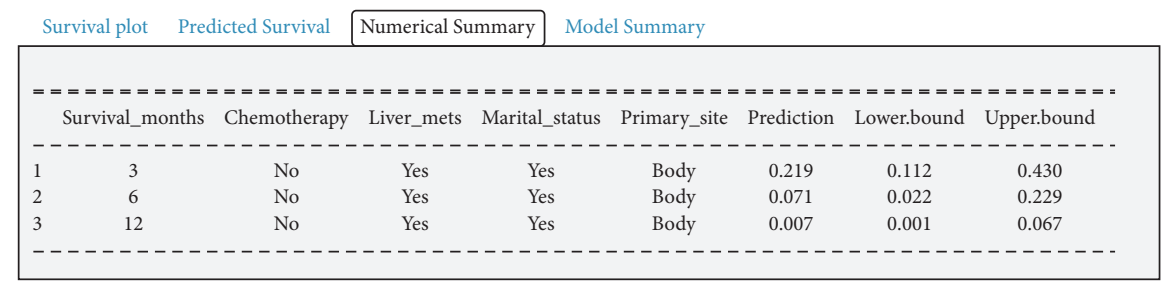

(d)

FIGURE 10: Web-based nomogram. (a) Operation page of web-based nomogram; (b) survival curve of the corresponding patient; (c) survival rates and 95\% confidence intervals at 3 months (black line), 6 months (blue line), and 12 months (red line); (d) the prediction accuracy of the corresponding patient.

We found that liver metastasis is an independent risk factor for OS and CSS in patients with GCLM. There are two possible reasons for this. First, the liver contains a rich blood supply, tumor metastasis is rapid, patients are already advanced when symptoms appear, and most of them miss the time of surgery. Second, for patients with GCLM and hepatocellular carcinoma (HCC), the prognosis is worse because the patients are lethargic and weak, and their immunity is reduced, typically when they develop complications associated with advanced HCC (such as jaundice, ascites, peritonitis, and hepatic encephalopathy). Chemotherapy was found to be an independent protective factor for OS and CSS. This result confirmed the importance and necessity of chemotherapy in patients with GCLM. The National Comprehensive Cancer Network guidelines clearly state that chemotherapy is recommended for the treatment of patients with unresectable or metastatic GC [33]. A study reported median OS times of 8.6 and 7.9 months for patients with advanced GC treated with cisplatin combined with S-1 (CS) versus cisplatin combined with 5-FU (CF) regimens, respectively $(P=0.02)$ [34]. Standardized chemotherapy not only relieves the patients' clinical symptoms but also prolongs the survival time. Hence, it is worthwhile to focus on the possibility of liver metastasis in patients with GCLM. To obtain an excellent prognosis, doctors could prefer chemotherapy for the clinical treatment of patients with GCLM. In addition, we incorporated marital status into our study. The results of this study showed that married patients with GCLM had better clinical prognosis than those who were unmarried. It has been shown that marriage plays a humanistic role during the treatment of oncology patients and that care plays a crucial role in influencing tumor progression [35].

However, there are some limitations to our study. First, although we have set strictly incorporated exclusion standards, the deletion of patients is missing and may cause statistical bias. Second, there is no detailed treatment information in the SEER database, such as specific chemotherapy modalities and surgical procedures. Third, the SEER database has limited coverage, and some important factors such as smoking, alcohol consumption, family history of tumor, and other factors that may affect patient prognosis were not assessed.

\section{Conclusions}

In conclusion, this study revealed that grade, liver metastasis, and chemotherapy were independent prognostic factors for OS, where the risk factors were grade and liver metastasis, and chemotherapy was a protective factor. Liver metastasis, primary site, chemotherapy, and marital status were independent prognostic factors for CSS, where liver metastasis and primary site were risk factors, and chemotherapy and marital status were protective factors. We created two easy-to-use visual web-based nomograms with several clinical and pathological factors to quantitatively predict OS and CSS in patients with GCLM. Moreover, our model may help physicians develop individualized postoperative follow-up strategies.
Abbreviations
AUC: Area under the curve
CSS: Cancer-specific survival
DCA: Decision curve analysis
GC: $\quad$ Gastric cancer
GCLM: Gastric cancer and lung metastasis
LM: $\quad$ Lung metastasis
OS: Overall survival
ROC: Receiver operating characteristic
SEER: Surveillance, Epidemiology, and End Results.

\section{Data Availability}

The dataset from the SEER database which was generated and/or analyzed during the current study is available in the SEER dataset repository (https://seer.cancer.gov/).

\section{Ethical Approval}

The study's approval was waived by the local ethics committee, as the SEER data were publicly available and deidentified.

\section{Conflicts of Interest}

The authors declare that there are no conflicts of interest with respect to the research, authorship, and/or publication of this article.

\section{Authors' Contributions}

Honghong Zheng and Zhehong Li contributed equally to this work. $\mathrm{HH} \mathrm{Z}$ and $\mathrm{ZH} \mathrm{L}$ conceived and designed the study. Honghong Zheng and Jianjun Li performed the 
literature search. Zhehong Li and Shuai Zheng generated the figures and tables. Jianjun Li and Shuai Zheng analyzed the data. Honghong Zheng and Zhehong Li wrote the manuscript, and Enhong Zhao critically reviewed the manuscript. Enhong Zhao supervised the study. All experiments and methods met the standards of relevant guidelines and regulations. All authors have read and approved the manuscript.

\section{Acknowledgments}

This study is funded by the 2020 Hebei Provincial Finance Department Grant for Outstanding Talents in Clinical Medicine (no. 2020397).

\section{References}

[1] F. Bray, J. Ferlay, I. Soerjomataram, R. L. Siegel, L. A. Torre, and A. Jemal, "Global cancer statistics 2018: GLOBOCAN estimates of incidence and mortality worldwide for 36 cancers in 185 countries," CA: A Cancer Journal for Clinicians, vol. 68, no. 6, pp. 394-424, 2018.

[2] Y.-J. Bang, Y.-W. Kim, H.-K. Yang et al., "Adjuvant capecitabine and oxaliplatin for gastric cancer after D2 gastrectomy (CLASSIC): a phase 3 open-label, randomised controlled trial," The Lancet, vol. 379, no. 9813, pp. 315-321, 2012.

[3] M. Sasako, S. Sakuramoto, H. Katai et al., "Five-year outcomes of a randomized phase III trial comparing adjuvant chemotherapy with S-1 versus surgery alone in stage II or III gastric cancer," Journal of Clinical Oncology, vol. 29, no. 33, pp. 4387-4393, 2011.

[4] K.-Y. Song, S.-M. Park, S.-N. Kim, and C.-H. Park, "The role of surgery in the treatment of recurrent gastric cancer," The American Journal of Surgery, vol. 196, no. 1, pp. 19-22, 2008.

[5] T. Cancer and T. Kajitani, "The general rules for the gastric cancer study in surgery and pathology part I," Japanese Journal of Surgery, vol. 11, no. 2, pp. 127-139, 1981.

[6] W.-M. Kang, Q.-B. Meng, J.-C. Yu, Z.-Q. Ma, and Z.-T. Li, "Factors associated with early recurrence after curative surgery for gastric cancer," World Journal of Gastroenterology, vol. 21, no. 19, pp. 5934-5940, 2015.

[7] G. Spolverato, A. Ejaz, Y. Kim et al., "Rates and patterns of recurrence after curative intent resection for gastric cancer: a United States multi-institutional analysis," Journal of the American College of Surgeons, vol. 219, no. 4, pp. 664-675, 2014.

[8] Y. Nakanishi, M. Ohara, H. Domen, T. Shichinohe, S. Hirano, and M. Ishizaka, "Differences in risk factors between patterns of recurrence in patients after curative resection for advanced gastric carcinoma," World Journal of Surgical Oncology, vol. 11, no. 1, pp. 98-8, 2013.

[9] J. Deng, H. Liang, D. Wang, D. Sun, Y. Pan, and Y. Liu, "Investigation of the recurrence patterns of gastric cancer following a curative resection," Surgery Today, vol. 41, no. 2, pp. 210-215, 2011.

[10] J. Y. Choi, T. K. Ha, and S. J. Kwon, "Clinicopathologic characteristics of gastric cancer patients according to the timing of the recurrence after curative surgery," Journal of Gastric Cancer, vol. 11, no. 1, pp. 46-54, 2011.

[11] R. C. Ye, S. H. Dong, S. H. Kong et al., "The value of palliative gastrectomy in gastric cancer with distant metastasis," Annals of Surgical Oncology, vol. 19, no. 4, pp. 1231-1239, 2012.
[12] T. Zheng, J. Jin, Y. Zhang, and L. Zhou, "Efficacy and safety of paclitaxel with or without targeted therapy as second-line therapy in advanced gastric cancer: a meta-analysis," Medicine (Baltimore), vol. 99, no. 25, Article ID e20734, 2020.

[13] Y. W. Choi, M. S. Ahn, G. S. Jeong et al., "The role of surgical resection before palliative chemotherapy in advanced gastric cancer," Scientific Reports, vol. 9, no. 1, Article ID 4136, 2019.

[14] Y.-K. Kang, N. Boku, T. Satoh et al., "Nivolumab in patients with advanced gastric or gastro-oesophageal junction cancer refractory to, or intolerant of, at least two previous chemotherapy regimens (ONO-4538-12, ATTRACTION-2): a randomised, double-blind, placebo-controlled, phase 3 trial," The Lancet, vol. 390, no. 10111, pp. 2461-2471, 2017.

[15] Y. Kobayashi, T. Fukui, S. Ito et al., "Pulmonary metastasectomy for gastric cancer: a 13-year single-institution experience," Surgery Today, vol. 43, no. 12, pp. 1382-1389, 2013.

[16] J. H. Kong, J. Lee, C.-A. Yi et al., "Lung metastases in metastatic gastric cancer: pattern of lung metastases and clinical outcome," Gastric Cancer, vol. 15, no. 3, pp. 292-298, 2012.

[17] M. A. Tursun and Z. W. Bin, "Analysis of prognostic factors affecting 118 cases of patients with lung metastases from gastric cancer," Chinese Journal of Cancer Control, vol. 26, no. 15, pp. 1107-1110, 2019.

[18] M. Chen, S. Li, W. Topatana et al., "Development and validation of a nomogram for predicting survival in gallbladder cancer patients with recurrence after surgery," Frontiers in Oncology, vol. 10, Article ID 537789, 2021.

[19] L.-J. Sun, A. Guan, W.-Y. Xu et al., " $\gamma$-glutamyl transferase-toplatelet ratio based nomogram predicting overall survival of gallbladder carcinoma," World Journal of Gastrointestinal Oncology, vol. 12, no. 9, pp. 1014-1030, 2020.

[20] J. Li, H. Yue, H. Yu, X. Lu, and X. Xue, "Development and validation of SIRT3-related nomogram predictive of overall survival in patients with serous ovarian cancer," Journal of Ovarian Research, vol. 12, no. 1, p. 47, 2019.

[21] W. Song, C.-g. Lv, D.-1. Miao et al., "Development and validation of a nomogram for predicting survival in patients with gastrointestinal stromal tumours," European Journal of Surgical Oncology, vol. 44, no. 10, pp. 1657-1665, 2018.

[22] T. Yang, Y. Wu, Y. Zuo, S. Fu, Z. Xu, and N. Yu, "Development and validation of prognostic nomograms and a webbased survival rate calculator for sarcomatoid renal cell carcinoma in pre- and post-treatment patients," Translational Andrology and Urology, vol. 10, no. 2, pp. 754-764, 2021.

[23] Y. W. Chung, D. S. Han, Y. K. Park et al., "Association of obesity, serum glucose and lipids with the risk of advanced colorectal adenoma and cancer: a case-control study in korea," Digestive and Liver Disease, vol. 38, no. 9, pp. 668-672, 2006.

[24] Y. Zhang, Y. Lin, J. Duan, K. Xu, M. Mao, and X. Wang, “A population-based analysis of distant metastasis in stage IV gastric cancer," Medical Science Monitor: International Medical Journal of Experimental and Clinical Research, vol. 26, Article ID e923867, 2020.

[25] N. Lui, P. Ronnie Tung Ping, and P. Roberta, "Biomarkers for predicting future metastasis of human gastrointestinal tumors," Cellular and Molecular Life Sciences: CMLS, vol. 70, no. 19, 2013.

[26] W. Zhang, G. Zhangyuan, J. Wang et al., "Effect of lymph nodes count in node-positive gastric cancer," Journal of Cancer, vol. 10, no. 23, pp. 5646-5653, 2019.

[27] Y. Waki, T. Irino, R. Makuuchi et al., "Impact of preoperative skeletal muscle quality measurement on long-term survival 
after curative gastrectomy for locally advanced gastric cancer," World Journal of Surgery, vol. 43, no. 12, pp. 3083-3093, 2019.

[28] C.-H. HSIEH, C.-K. WEI, W.-Y. YIN et al., "Vascular invasion affects survival in early hepatocellular carcinoma," Molecular and clinical oncology, vol. 3, no. 1, pp. 252-256, 2015.

[29] S.-W. Suh, K.-W. Lee, J.-M. Lee et al., "Prediction of aggressiveness in early-stage hepatocellular carcinoma for selection of surgical resection," Journal of Hepatology, vol. 60, no. 6, pp. 1219-1224, 2014.

[30] Y. Tomimaru, H. Eguchi, S. Marubashi et al., "Equivalent outcomes after anatomical and non-anatomical resection of small hepatocellular carcinoma in patients with preserved liver function," Digestive Diseases and Sciences, vol. 57, no. 7, pp. 1942-1948, 2012.

[31] C. Hu, J. Yang, Z. Huang et al., "Diagnostic and prognostic nomograms for bone metastasis in hepatocellular carcinoma," BMC Cancer, vol. 20, no. 1, p. 494, 2020.

[32] C. Neuzillet, A. Casadei Gardini, B. Brieau et al., "Prediction of survival with second-line therapy in biliary tract cancer: actualisation of the AGEO CT2BIL cohort and European multicentre validations," European Journal of Cancer, vol. 111, pp. $94-106,2019$.

[33] J. A. Ajani, T. A. D’Amico, K. Almhanna et al., “Gastric cancer, version 3.2016, NCCN clinical practice guidelines in oncology," Journal of the National Comprehensive Cancer Network: Journal of the National Comprehensive Cancer Network, vol. 14, no. 10, pp. 1286-1312, 2016.

[34] J. A. Ajani, W. Rodriguez, G. Bodoky et al., "Multicenter phase III comparison of cisplatin/S-1 with cisplatin/infusional fluorouracil in advanced gastric or gastroesophageal adenocarcinoma study: the FLAGS trial," Journal of Clinical Oncology, vol. 28, no. 9, pp. 1547-1553, 2010.

[35] J. J. Jin, W. Wang, F. X. Dai et al., "Marital status and survival in patients with gastric cancer," Cancer Medicine, vol. 5, no. 8, pp. 1821-1829, 2016. 